\section{Clinical applications of donor lymphocyte infusion from an HLA-haploidentical donor: consensus recommendations from the Acute Leukemia Working Party of the EBMT}

\author{
Bhagirathbhai Dholaria, ${ }^{1}$ Bipin N. Savani, ${ }^{1}$ Myriam Labopin, ${ }^{2}$ Leo Luznik, ${ }^{3}$ Annalisa \\ Ruggeri, ${ }^{4}$ Stephan Mielke, ${ }^{5}$ Monzr M. Al Malki, ${ }^{6}$ Piyanuch Kongtim, ${ }^{7}$ Ephraim \\ Fuchs, ${ }^{8}$ Xiao-Jun Huang, ${ }^{9}$ Franco Locatelli, ${ }^{10}$ Franco Aversa, ${ }^{11}$ Luca Castagna, ${ }^{12}$ \\ Andrea Bacigalupo, ${ }^{13}$ Massimo Martelli, ${ }^{14}$ Didier Blaise,${ }^{15}$ Patrick Ben Soussan, ${ }^{16}$ \\ Yolande Arnault, ${ }^{17}$ Rupert Handgretinger, ${ }^{18}$ Denis-Claude Roy, ${ }^{19}$ Paul O'Donnell, ${ }^{20}$ \\ Asad Bashey, ${ }^{21}$ Scott Solomon, ${ }^{21}$ Rizwan Romee, ${ }^{22}$ Philippe Lewalle ${ }^{23}$ Jorge \\ Gayoso, ${ }^{24}$ Michael Maschan, ${ }^{25}$ Hillard M. Lazarus, ${ }^{26}$ Karen Ballen, ${ }^{27}$ Sebastian \\ Giebel, ${ }^{28}$ Frederic Baron, ${ }^{29}$ Fabio Ciceri, ${ }^{30}$ Jordi Esteve, ${ }^{31}$ Norbert-Claude Gorin, ${ }^{32}$ \\ Alexandros Spyridonidis, ${ }^{33}$ Christoph Schmid, ${ }^{34}$ Stefan O. Ciurea, ${ }^{35}$ Arnon Nagler ${ }^{36}$ \\ and Mohamad Mohty ${ }^{37}$
}

${ }^{1}$ Department of Hematology-Oncology, Vanderbilt University Medical Center, Nashville, TN, USA: ${ }^{2}$ Department of Haematology and EBMT Paris study office / CEREST-TC, Saint Antoine Hospital, Paris, France; ${ }^{3}$ Department of Oncology Hematologic Malignancies, Johns Hopkins University School of Medicine, Baltimore, MD, USA; ${ }^{4}$ Department of Pediatric Hematology/Oncology and Cell and Gene Therapy, IRCCS Ospedale Pediatrico Bambino Gesù, Rome, Italy; ${ }^{5}$ Department of Laboratory Medicine, CAST, Karolinska Institutet and University Hospital, Stockholm, Sweden; ${ }^{6}$ Department of Hematology and Hematopoietic Cell Transplantation, City of Hope National Medical Center, Duarte, CA, USA; 고em Cell Transplant and Cellular Therapy, Thammasat University, Pathumthani, Thailand; ${ }^{8}$ Johns Hopkins University, Sidney Kimmel Comprehensive Cancer Center, Baltimore, MD, USA; 'Peking University People's Hospital, Peking University Institute of Hematology, Beijing China; ${ }^{10}$ Department of Pediatric Hematology/Oncology, IRCCS Ospedale Pediatrico Bambino Gesù, Rome, Sapienza, University of Rome, Italy; ${ }^{11}$ Department of Medicine and Surgery, University of Parma, Parma, Italy; ${ }^{12}$ Instituto Clinico Humanitas, Rozzano Milano, Italy; ${ }^{13}$ Fondazione Policlinico Universitario Gemelli IRCCS, Università Cattolica del Sacro Cuore, Rome, Italy; ${ }^{14}$ University of Perugia, Sant'Andrea Delle Fratte, Perugia Italy; ${ }^{15}$ Department of Hematology, Institut Paoli Calmettes, Marseille France; ${ }^{16}$ Department of Clinical Psychology, Paoli-Calmettes Institute, Marseille, France; ${ }^{17}$ Institut Paoli-Calmette, département de psychologie clinique, Marseille, France; ${ }^{18}$ Department of Hematology and Oncology, University Children's Hospital Tübingen, Tübingen Germany; ${ }^{19}$ Division of Hematology and Medical Oncology, Hospital Maisonneuve-Rosemont, Montreal, QC, Canada; ${ }^{20} \mathrm{Hematology-Oncology,}$ Massachusetts General Hospital, Harvard Medical School, Boston, MA, USA; ${ }^{21}$ Blood and Marrow Transplant Program at Northside Hospital, Atlanta, GA, USA; ${ }^{22}$ Dana Farber Cancer Institute, Harvard Medical School, Boston, MA, USA; ${ }^{23} \mathrm{Hematology} \mathrm{Department,} \mathrm{Institut} \mathrm{Jules}$ Bordet, Université Libre de Bruxelles, Brussels, Belgium; ${ }^{24} \mathrm{HGU}$ Gregorio Marañón, Instituto de Investigación Sanitaria Gregorio Marañón, Madrid, Spain; ${ }^{25}$ Oncology and immunology, Dmitriy Rogachev National Medical Center of pediatric hematology, Moscow, Russia; ${ }^{26}$ Adult Hematologic Malignancies \& Stem Cell Transplant Section, University Hospitals Seidman Cancer Center, Cleveland, OH, USA; ${ }^{27}$ Division of hematology/oncology, University of Virginia Health System, Charlottesville, VA, USA; ${ }^{28}$ Dept. of Bone Marrow Transplantation and OncoHematology, Maria Sklodowska-Curie Institute - Oncology Center, Gliwice Branch, Gliwice, Poland; ${ }^{29}$ Laboratory of Hematology, University of Liège, Liège, Belgium; ${ }^{30} \mathrm{Hematology}$ and Bone Marrow Transplantation Unit, San Raffaele Scientific Institute, Milano Italy; ${ }^{31} \mathrm{Hematology}$ department, Hospital Clínic de Barcelona, Barcelona Spain; ${ }^{32}$ Service d'hématologie et thérapie cellulaire Centre international greffes APHP-EBMT-INCa Hospital, Saint Antoine Hospital, Paris France; ${ }^{33}$ Bone Marrow Transplantation Unit and CBMDP Donor Center, University Hospital of Patras, Patras, Greece; ${ }^{34}$ Department of Hematology and Oncology, Klinikum Augsburg, Augsburg, Germany; ${ }^{35}$ Stem Cell Transplant and Cellular Therapy, The University of Texas MD Anderson Cancer Center, Houston, TX, USA; ${ }^{36}$ Chaim Sheba Medical Center, Tel Aviv University, Tel-Hashomer, Israel and EBMT ALWP office, Saint Antoine Hospital , Paris, France and ${ }^{37}$ Service d'Hématologie Clinique et Thérapie Cellulaire, Hôpital Saint-Antoine, AP-HP, Sorobonne University, and INSERM UMRs 938, Paris, France

\section{ABSTRACT}

$\mathrm{D}$ onor lymphocyte infusion has been used in the management of relapsed hematologic malignancies after allogeneic hematopoietic cell transplantation. It can eradicate minimal residual disease or be used to rescue a hematologic relapse, being able to induce durable remissions in a subset of patients. With the increased use of haploidentical
Ferrata Storti Foundation

\author{
Haematologica 2020 \\ Volume 105(1):47-58
}

\section{Correspondence:}

BHAGIRATHBHAI DHOLARIA

bhagirathbhai.r.dholaria@vumc.org

Received: February 17, 2019.

Accepted: September 19, 2019.

Pre-published: September 19, 2019.

doi:10.3324/haematol.2019.219790

Check the online version for the most updated information on this article, online supplements, and information on authorship \& disclosures: www.haematologica.org/content/105/1/47

\section{(C)2020 Ferrata Storti Foundation}

Material published in Haematologica is covered by copyright. All rights are reserved to the Ferrata Storti Foundation. Use of published material is allowed under the following terms and conditions:

https://creativecommons.org/licenses/by-nc/4.0/legalcode. Copies of published material are allowed for personal or internal use. Sharing published material for non-commercial purposes is subject to the following conditions:

https://creativecommons. org/licenses/by-nc/4.0/legalcode, sect. 3. Reproducing and sharing published material for commercial purposes is not allowed without permission in writing from the publisher. 
hematopoietic cell transplantation, there is renewed interest in the use of donor lymphocytes to either treat or prevent disease relapse post transplant. Published retrospective and small prospective studies have shown encouraging results with therapeutic donor lymphocyte infusion in different haploidentical transplantation platforms. In this consensus paper, finalized on behalf of the Acute Leukemia Working Party of the European Society for Blood and Marrow Transplantation, we summarize the available evidence on the use of donor lymphocyte infusion from haploidentical donor, and provide recommendations on its therapeutic, pre-emptive and prophylactic use in clinical practice.

\section{Introduction}

Allogeneic hematopoietic cell transplantation (alloHCT) remains an important therapeutic option for a wide number of both hematologic malignancies and nonhematologic disorders. With improvements in conditioning regimens, graft-versus-host disease (GvHD) prophylaxis and supportive care, leading to a reduced risk of transplant-related mortality, disease relapse has become the foremost cause of mortality after allo-HCT. The cumulative incidence of relapse (CIR) after allo-HCT for acute leukemia can be as high as $40-50 \%$ with only 10 $15 \%$ long-term survival in patients experiencing leukemia recurrence..$^{1.3}$ Strategies aimed at preventing and/or treating disease relapse have the greatest potential to improve transplant outcomes. Donor-lymphocyte infusion (DLI) has an established role in the management of disease relapse after allo-HCT. Unmanipulated DLI is a form of immunotherapy, which can induce durable remissions by enhancing the graft-versus-tumor (GvT) effect. ${ }^{4 \cdot 6}$ Efficacy of DLI varies by type and burden of the disease. DLI is more effective in chronic myeloid leukemia (CML), leading to complete remission (CR) in $70-80 \%$ of patients in hematologic or cytogenetic CML relapse, whereas less than $40 \%$ of acute leukemia patients respond to DLI., $\mathrm{A}$ study by Schmid et al. using the European Society for Blood and Marrow Transplantation (EBMT) registry showed DLI was associated with improved survival of patients with AML in the first hematologic relapse after allo-HCT, but 2-year overall survival (OS) was only $15 \%$ if DLI was given in the setting of active disease. ${ }^{10}$ Preemptive DLI for mixed chimerism or molecular disease relapse and prophylactic DLI for high-risk hematologic malignancies have also been studied with promising results in the setting of human leukocyte antigen (HLA)matched allo-HCT.11-13

Allo-HCT from an HLA-haploidentical related donor (haplo-HCT) has emerged as a suitable alternative for those patients who need an allograft but who lack an HLA-matched related or unrelated donor. ${ }^{14,15}$ Several Tcell depleted and T-cell replete haploidentical transplant strategies are applied today. In T-cell replete haploidentical stem cell transplantation, the use of post-transplant cyclophosphamide (PTCy) has rapidly increased across the globe due to its logistical simplicity and efficacy. ${ }^{2}$ Another T-cell replete haplo-HCT platform is granulocyte colony stimulating factor (GCSF)-antithymocyte globulin (ATG)-based or "GIAC" protocol" [GCSF-stimulation of the donor; intensified immunosuppression through posttransplantation cyclosporine (CSA), mycophenolate mofetil (MMF), and short-course methotrexate (MTX); anti-thymocyte globulin (ATG); and combination of peripheral blood stem cells (PBSC) and bone marrow (BM) allografts] initially developed at the Peking University. ${ }^{16}$ Today's T-cell depleted strategies derive from the mega-dose $\mathrm{CD} 34^{+}$protocol developed at the Perugia University ${ }^{17}$ and represent a historical standard in T-cell depletion. From this platform, several other T-cell depleted strategies have evolved, such as $\mathrm{CD} 3 / \mathrm{CD} 19$ cell depletion ${ }^{18}$ and $\alpha-\beta-T / C D 19-B$ cell depletion. ${ }^{19}$ The adoptive transfer of selectively allo-depleted ${ }^{20}$ or gene-modified T cells with a suicide switch ${ }^{21,22}$ after T-cell depleted transplantation have further optimized this transplant form and are now being investigated in large randomized trials in comparison with PTCy.

Haplo-HCT with PTCy has shown comparable clinical outcomes to matched unrelated donor allo-HCT in retrospective analyses with a significantly lower risk of chronic GvHD in myeloid and lymphoid malignancies, regardless of whether the graft was obtained from BM or mobilized PBSC..$^{23-26}$ There is concern that DLI from a haploidentical donor (haplo-DLI) may pose an increased risk for GvHD, given the higher degree of HLA disparity between the donor and recipient. However, a greater HLA-disparity may also be beneficial in promoting a stronger GvT effect. ${ }^{27}$ Another advantage is that a related haploidentical donor is, in most cases, readily available and collection is faster than a registry-based unrelated donor. While the experience with haplo-DLI is limited, and there are many uncertainties around its clinical application, it can be a powerful tool to manage a disease relapse after haplo-HCT. Nonetheless, it should be emphasized that haplo-DLI after T-cell depleted transplantation without full immune reconstitution may have very different effects than after T-cell replete transplantation and may require completely different dosing strategies. Therefore, in the absence of data from prospective clinical trials, general recommendations cannot be made.

In this review, we summarize the published experience with haplo-DLI and provide recommendations regarding its use in various clinical settings (therapeutic vs. preemptive vs. prophylactic DLI), use of chemotherapy before DLI, optimal cell dose, and concurrent immunosuppression management. Newer strategies using cellular engineering, donor-derived natural killer (NK) cells and pharmacological immunomodulation are also discussed.

\section{Therapeutic haplo-donor-lymphocyte infusion: hematologic relapse}

Previously published retrospective studies have suggested that outcomes of haplo-DLI in patients with hematologic relapse are comparable to standard DLI from an HLA-matched donor. The incidence of DLI-associated GvHD also appears to be similar regardless of donor type ${ }^{28-30}$ Possible explanations, at least when used late post transplant, are the use of lower cell dose and presence of donor-derived tolerogenic cells in the recipient, 
which may reduce their alloreactivity and, thus, the risk of GvHD. The type of haplo-HCT protocol may influence outcomes of subsequent haplo-DLI. The current therapeutic haplo-DLI experience is limited to haploHCT/PTCy or a 'GCSF-ATG-based protocol'. Table 1 summarizes the published studies using DLI from a haploidentical donor.

\section{Therapeutic donor-lymphocyte infusion in T-cell replete haplo-hematopoietic cell transplantation}

Zeidan et al. retrospectively reported results of haploDLI in 40 patients [minimal residual disease (MRD)/loss of chimerism (LOC): $n=5$; hematologic relapse: $n=35$ ] after a haplo-HCT/PTCy with BM graft. At the median follow up of seven months, CR was achieved in $30 \%$ of patients (CR after a hematologic relapse: $26 \%$ ) and acute $\mathrm{GvHD}(\mathrm{aGvHD})$ occurred in $25 \%$ of them. At time of last follow up, six patients were alive in CR for over a year after the intervention. The cell dose in most DLI was $1 \times 10^{6} \mathrm{CD}^{+}$cells $/ \mathrm{kg}$ and the majority of patients received cytoreductive chemotherapy before DLI. ${ }^{28}$ Subsequently, two similar reports showed that haplo-DLI after chemotherapy successfully resulted in CR in approximately $30 \%$ of the patients with a subset of long-term survivors. ${ }^{29,30}$ The incidence of grade 2-4 aGvHD was approximately $30 \%$, and only $5 \%$ of patients developed grade 3-4 aGvHD. No patient ( $0 \%$ ) developed extensive chronic GvHD (cGvHD). ${ }^{29,30}$ None of these studies used immunosuppression for GvHD prophylaxis after haploDLI. Disease responses and GvHD rates were comparable between patients who received BM versus a PBSC graft. ${ }^{30}$ Patients with relapsed Hodgkin lymphoma appear to have relatively better disease responses to haplo-DLI compared to those with acute leukemia (40\% vs. 33\%). ${ }^{29}$ In a smaller retrospective study $(n=21)$ published by Goldsmith et al., the authors showed that patients with extra-medullary disease relapse had a better relapse-free survival (RFS) compared to those with marrow relapse (4month RFS $43 \%$ vs. $8 \%$ ). ${ }^{30}$ The group at Peking University has developed a haplo-DLI protocol using GCSF-primed peripheral blood progenitor cells (GBPC) with short-term immunosuppression. They have used a higher cell dose $\left(1 \times 10^{7}\right.$ to $1 \times 10^{8} \mathrm{CD3}^{+}$cells $\left./ \mathrm{kg}\right)$ than that used in haplo-DLI in the setting of allo-HCT/PTCy $\left(1 \times 10^{5}\right.$ to $1 \times 10^{6} \mathrm{CD}^{+}$ cells $/ \mathrm{kg}) .{ }^{31-37}$ An earlier prospective study using GBPC without immunosuppression resulted in a high incidence of severe GvHD (grade 3-4 aGvHD 30\%, extensive cGvHD 30\%), resulting in 2-year disease-free survival (DFS) of $40 \%$ and non-relapse mortality (NRM) of $25 \% .{ }^{38}$ Subsequent studies used cytoreductive chemotherapy before GBPC infusion (chemo-DLI) followed by GvHD prophylaxis with low-dose weekly MTX or CSA for 6-8 weeks. A retrospective report by Yan et al. on 55 patients with relapsed acute leukemia showed 3-year DFS, NRM, and OS of $24 \%, 13 \%$, and $25 \%$, respectively. A total of $76 \%$ of patients achieved CR (MRD negative CR: $55 \%$ ). ${ }^{39}$ Relapse after achieving CR following chemo-DLI was a major problem, resulting in poor long-term survival. In spite of the limitations of cross-study comparison, outcomes of chemo-GBPC infusion with short-course immunosuppression are comparable to unmanipulated haplo-DLI after haplo-HCT/PTCy.

\section{Pre-emptive haplo-donor-lymphocyte infusion: minimal residual disease, mixed-donor chimerism}

\section{Impact of minimal residual disease and mixed- chimerism on haplo-hematopoietic cell transplantation outcomes}

Strategies are being explored to reliably predict the risk of disease relapse after an allo-HCT in the hope of implementing pre-emptive treatments. The presence of MRD before or after allo-HCT is associated with significantly increased risk of relapse and reduced survival in both acute lymphoblastic leukemia (ALL) and acute myeloid leukemia (AML). ${ }^{40,41}$ Canaani et al. looked at pre-haploHCT MRD positivity in AML patients and showed its negative correlation with leukemia-free survival. ${ }^{42}$ Low donor T-cell chimerism [mixed-chimerism (MC)] after an allo-HCT is also associated with poor donor-derived immune reconstitution and increased risk of disease relapse, especially after myeloablative conditioning. In patients with AML/MDS who underwent myeloablative allo-HCT, donor T-cell chimerism $<85 \%$ at day (d)+90 and $d+120$ was associated with increased risk of 3-year disease progression $(\mathrm{HR}=2.1, P=0.04){ }^{43}$ Koreth et al. reported that $d+100$ total donor chimerism $<90 \%$ was associated with increased risk of relapse $(\mathrm{HR}=2.54$, $P<0.001)$ and lower $\mathrm{OS}(\mathrm{HR}=1.50, P=0.009)$ in patients after a reduced-intensity allo-HCT. ${ }^{44}$ Pre-emptive DLI from a full matched donor for MRD and MC appears to be safe and effective in improving disease-specific outcomes. ${ }^{45,46}$

\section{Pre-emptive donor-lymphocyte infusion in T-cell replete haplo-hematopoietic cell transplantation}

In the previously mentioned retrospective study by Zeidan et al., 3 of 4 patients who received haplo-DLI for MRD entered CR. ${ }^{28}$ Similarly, other reports showed higher response rates in patients who received haplo-DLI for $\mathrm{MRD}$ or $\mathrm{MC}$ compared to the administration at the time of hematologic relapse. ${ }^{29,30}$ Yan et al. reported comparative outcomes of prospective studies of standard-risk acute leukemia and MDS patients with persistent MRD after allo-HCT (haploidentical donor, $n=29$; matched donor, $\mathrm{n}=27$ ), who received low-dose interleukin-2 (IL-2) or preemptive DLI. The latter was associated with reduced 3 year CIR compared to IL-2 alone (28\% vs. $64 \%$; $P=0.001) .{ }^{31}$ In another retrospective study by Mo et al., 101 patients (haplo-HCT, $n=56$ ) received chemo-DLI for persistent MRD after an allo-HCT. Three-year CIR, NRM, and OS were $40 \%, 10 \%$, and $52 \%$, respectively. Patients who cleared their MRD within 30 days after preemptive chemo-DLI had lower relapse rates compared to those with persistent MRD beyond 30 days (20\% vs. $47 \% ; P=0.001) .{ }^{36}$ It should be noted that the published data on pre-emptive haplo-DLI for MC is limited to a few patients $^{28,30}$ and further studies are needed to establish its role in preventing disease relapse.

It is important to monitor for MRD after allo-HCT as DLI is probably more effective when administered for MRD only compared overt hematologic relapse. ${ }^{47}$ Retrospective studies have shown that persistent MRD post-transplant is associated with high relapse rate and poor outcomes, ${ }^{48}$ and the eradication of MRD improves survival. ${ }^{46}$ Comparative studies are needed between DLI and other systemic therapies in order to develop disease- 
Table 1. Donor-lymphocyte infusion from a haploidentical donor.

\begin{tabular}{|c|c|c|c|c|c|c|c|c|c|c|}
\hline $\begin{array}{l}\text { T-cell depleted } \\
\text { haplo-HCT }\end{array}$ & $\begin{array}{l}\text { N. of patients } \\
\text { (prospective/ } \\
\text { retrospective) }\end{array}$ & Diagnosis & $\begin{array}{l}\text { Indication } \\
\text { for DLI }\end{array}$ & $\begin{array}{l}\text { Treatments } \\
\text { before DLI }\end{array}$ & $\begin{array}{c}\text { CD3 }^{+} \\
\text {dose } / \mathrm{kg}\end{array}$ & $\begin{array}{l}\text { N. of DLI } \\
\text { (median) }\end{array}$ & $\begin{array}{l}\text { Disease } \\
\text { response }\end{array}$ & $\begin{array}{c}\text { Rate of } \\
\text { GvHD }\end{array}$ & Survival & Notes \\
\hline $\begin{array}{l}\text { Lewalle et al. } \\
(2003)^{65}\end{array}$ & $\begin{array}{c}12 \\
\text { (prospective) }\end{array}$ & $\begin{array}{c}\mathrm{AML}=5 \\
\mathrm{ALL}=3 \\
\mathrm{CML}=1 \\
\text { Other }=3\end{array}$ & Prophylactic $=12$ & None & $1-4 \times 10^{4}$ & & & $58 \%$ & $\begin{array}{c}1-\mathrm{yr} \text { RFS }=50 \% \\
1-\mathrm{yr} \text { OS }=50 \% \\
\mathrm{NRM}=0 \%\end{array}$ & $\begin{array}{c}\text { T-/B-cell } \\
\text { depleted graft }\end{array}$ \\
\hline $\begin{array}{l}\text { Dodero et al. } \\
(2009)^{62}\end{array}$ & $\begin{array}{c}23 \\
\text { (prospective) }\end{array}$ & $\begin{array}{c}\text { Lymphoma } \\
\text { CLL } \\
\text { ALL } \\
\text { MM } \\
\text { AML }\end{array}$ & Prophylactic-23 & None & $2-15 \times 10^{4}$ & 2 & NA & $\begin{array}{c}\text { aGvHD }=26 \% \\
\text { (grade } 3-4=9 \% \text { ) } \\
\text { cGvHD }=15 \% \\
\text { (extensive }=12 \% \text { ) }\end{array}$ & $\begin{array}{c}2 \text {-yr } \mathrm{PFS}=45 \% \\
2 \text {-yr OS=44\% } \\
\mathrm{NRM}=26 \%\end{array}$ & $\begin{array}{l}\text { CD8 }{ }^{+} \text {T-cell } \\
\text { depleted DLI }\end{array}$ \\
\hline $\begin{array}{l}\text { Martelli et al. } \\
(2014)^{61}\end{array}$ & $\begin{array}{c}43 \\
\text { (prospective) }\end{array}$ & $\begin{array}{l}\mathrm{AML}=33 \\
\mathrm{ALL}=10\end{array}$ & Prophylactic-43 & None & $\begin{array}{l}\mathrm{T}_{\text {reg }}=2.5 \times 10^{6} \pm 1.1 \\
\mathrm{~T}_{\text {con }}=1.1 \times 10^{6} \pm 0.6\end{array}$ & 1 & NA & $\begin{array}{c}\text { aGvHD } \\
\text { (grade } \geq 2 \text { ) }=15 \% \\
\text { cGvHD }=16 \% \\
\text { (extensive }=7 \% \text { ) }\end{array}$ & $\begin{array}{l}\text { CI NRM }=40 \% \\
46-\mathrm{m} \text { relapse } \\
\quad=5 \%\end{array}$ & $\begin{array}{l}\mathrm{T}_{\text {reg }} \text { on day- } 4 \text {, } \\
\mathrm{T}_{\text {con }} \text { on day } 0\end{array}$ \\
\hline $\begin{array}{l}\text { Gilman et al. } \\
(2018)^{60}\end{array}$ & $\begin{array}{c}34 \\
\text { (prospective) }\end{array}$ & $\begin{array}{c}\text { AML/MDS }=13 \\
\text { ALL }=10 \\
\text { Other }=11\end{array}$ & Prophylactic-34 & None & $3-5 \times 10^{4}$ & 1 & NA & $\begin{array}{c}\text { aGvHD } \\
\text { (grade } 3-4 \text { ) }=4 \% \\
\text { cGvHD=16\% } \\
\text { (extensive=7\%) }\end{array}$ & $\begin{array}{c}2 \text {-yr OS=63\% } \\
2 \text {-yr NRM=25\% }\end{array}$ & $\begin{array}{c}\text { Post-DLI-GvHD } \\
\mathrm{ppx}=100 \%\end{array}$ \\
\hline
\end{tabular}

\section{T-cell replete}

haplo-HCT

\begin{tabular}{|c|c|c|c|c|c|c|c|c|c|c|}
\hline $\begin{array}{l}\text { Or et al. } \\
(2006)^{106}\end{array}$ & $\begin{array}{c}28 \\
\text { (haplo-26,MMUD-2) } \\
\text { (retrospective) }\end{array}$ & $\begin{array}{c}\text { AML }=12 \\
\text { ALL }=7 \\
\text { CML }=5 \\
\text { Other }=3\end{array}$ & $\begin{array}{l}\text { Prophylactic-6 } \\
\text { Therapeutic- } 22\end{array}$ & None & $1 \times 10^{2}$ to $15 \times 10^{8}$ & $1-7(1)$ & $\mathrm{CR}=18 \%$ & $46 \%$ & $\mathrm{NRM}=11 \%$ & \\
\hline $\begin{array}{l}\text { Huang et al. } \\
(2007)^{38 *}\end{array}$ & $\begin{array}{c}20 \\
\text { (prospective) }\end{array}$ & $\begin{array}{l}\mathrm{AML}=7 \\
\mathrm{ALL}=10 \\
\mathrm{CML}=3\end{array}$ & Therapeutic $=20$ & $\begin{array}{r}\mathrm{CT}=9 \\
\mathrm{TKI}=2\end{array}$ & $0.07-4.39 \times 10^{8}$ & $1-3(1)$ & $\mathrm{CR}=75 \%$ & $\begin{array}{c}\text { aGvHD }=55 \% \\
\text { (grade } 3-4=30 \% \text { ) } \\
\text { cGvHD }=64 \% \\
\text { (extensive }=30 \% \text { ) }\end{array}$ & $\begin{array}{c}2-\mathrm{yr} \text { DFS }=40 \% \\
\mathrm{NRM}=25 \%\end{array}$ & $\begin{array}{c}\text { Post-DLI-GvHD } \\
\text { ppx }=55 \%\end{array}$ \\
\hline $\begin{array}{l}\text { Yan et al. } \\
(2012)^{31 *}\end{array}$ & $\begin{array}{l}56 \\
\text { (haplo-29, matched } \\
\text { related-26, MUD-1) } \\
\text { (prospective) }\end{array}$ & $\begin{array}{l}\mathrm{AML}=32 \\
\mathrm{ALL}=21 \\
\mathrm{MDS}=3\end{array}$ & $\begin{array}{c}\text { Pre-emptive }=56 \\
\text { (MRD) }\end{array}$ & $\mathrm{CT}=32$ & $0.75-2 \times 10^{8}$ & 1 & NA & $\begin{array}{c}\text { aGvHD }=31 \% \\
\text { (grade } 3-4=8 \% \text { ) } \\
\text { cGvHD=43\% } \\
\text { (extensive }=34 \% \text { ) }\end{array}$ & $\begin{array}{c}3 \text {-yr DFS }=56 \% \\
3 \text {-yr OS=58\% } \\
3 \text {-yr NRM }=14 \%\end{array}$ & $\begin{array}{c}\text { Post-DLI-GvHD } \\
\text { ppx }=100 \%\end{array}$ \\
\hline $\begin{array}{l}\text { Yan et al. } \\
(2012)^{69 *}\end{array}$ & $\begin{array}{c}124 \\
\text { (retrospective) }\end{array}$ & $\begin{array}{l}\mathrm{AML}=49 \\
\mathrm{ALL}=59 \\
\mathrm{MDS}=5 \\
\mathrm{CML}=11\end{array}$ & $\begin{array}{c}\text { Prophylactic }=74 \\
\text { Therapeutic/ } \\
\text { pre-emptive= } \\
50 \text { (MRD) pt }\end{array}$ & $\begin{array}{l}\text { CT }=27 \\
(\mathrm{MRD}+) \\
\text { All relapsed } \\
\text { ts received CT. }\end{array}$ & $\begin{array}{l}0.13-2.11 \times 10^{8} \\
\text { T. }\end{array}$ & $1-4(1)$ & NA & $\begin{array}{c}\mathrm{aGvHD}=53 \% \\
\text { (grade } 3-4=28 \% \text { ) }\end{array}$ & $\begin{array}{c}2 \text {-yr CIR }=35 \% \\
2 \text {-yr OS }=47 \% \\
\text { 2-yr NRM }=34 \% \\
\text { In disease relapse, } \\
2 \text {-yr CIR }=45 \% \\
2 \text {-yr OS }=19 \% \\
2 \text {-yr NRM }=37 \%\end{array}$ & $\begin{array}{l}\text { Post-DLI-GvHD } \\
\text { ppx }=100 \%\end{array}$ \\
\hline $\begin{array}{l}\text { Wang et al. } \\
(2012)^{66 *}\end{array}$ & $\begin{array}{c}61 \\
\text { (retrospective) }\end{array}$ & $\begin{array}{l}\mathrm{AML}=42 \\
\mathrm{ALL}=19\end{array}$ & Prophylactic $=61$ & $\mathrm{CT}=0 \%$ & $0.9-7.2 \times 10^{8}$ & 1 & NA & $\begin{array}{c}\text { aGvHD=48\% } \\
\text { (grade } 3-4=10 \% \text { ) } \\
\text { cGvHD=39\% } \\
\text { (extensive }=31 \% \text { ) }\end{array}$ & $\begin{array}{c}3 \text {-yr DFS=22\% } \\
3 \text {-yr OS=31\% } \\
2 \text {-yr NRM=38\% }\end{array}$ & $\begin{array}{c}\text { Post-DLI-GvHD } \\
\text { ppx }=100 \%\end{array}$ \\
\hline $\begin{array}{l}\text { Yan et al. } \\
(2013)^{33 *}\end{array}$ & $\begin{array}{c}50 \\
\text { (retrospective) }\end{array}$ & $\begin{array}{l}\mathrm{AML}=29 \\
\mathrm{ALL}=21\end{array}$ & Therapeutic $=50$ & $\mathrm{CT}=100 \%$ & $0.11-2.07 \times 1^{08}$ & NA & $\mathrm{CR}=64 \%$ & $\begin{array}{c}\text { aGvHD }=66 \% \\
\text { (grade } 3-4=40 \% \text { ) } \\
\text { cGvHD }=44 \% \\
\text { (extensive }=42 \% \text { ) }\end{array}$ & $\begin{array}{c}1-\mathrm{yr} \text { DFS }=36 \% \\
1-\mathrm{yr} \text { OS }=36 \% \\
1-\mathrm{yr} \mathrm{NRM}=14 \%\end{array}$ & $\begin{array}{c}\text { Post-DLI-GvHD } \\
\text { ppx }=100 \%\end{array}$ \\
\hline $\begin{array}{l}\text { Yan et al. } \\
(2015)^{39 *}\end{array}$ & $\begin{array}{c}55 \\
\text { (retrospective) }\end{array}$ & $\begin{array}{l}A M L=18 \\
A L L=23\end{array}$ & Therapeutic $=55$ & $\mathrm{CT}=100 \%$ & $0.19-0.74 \times 10^{8}$ & NA & $\begin{array}{c}\mathrm{CR}=76 \% \\
\text { MRD negative } \\
\text { CR }=55 \%\end{array}$ & $\begin{array}{c}\text { aGvHD=44\% } \\
\text { (grade } 3-4=11 \% \text { ) } \\
\text { cGvHD=49\% } \\
\text { (extensive }=42 \% \text { ) }\end{array}$ & $\begin{array}{c}3 \text {-yr DFS }=24 \% \\
3 \text {-yr OS=25\% } \\
3 \text {-yr NRM }=13 \%\end{array}$ & $\begin{array}{c}\text { Post-DLI-GvHD } \\
\text { ppx }=100 \%\end{array}$ \\
\hline $\begin{array}{l}\text { Mo et al. } \\
(2016)^{36 *}\end{array}$ & $\begin{array}{c}101 \\
(\text { haplo=58) } \\
\text { (retrospective) }\end{array}$ & $\begin{array}{c}\text { AML/MDS }=69 \\
\text { ALL }=32\end{array}$ & $\begin{array}{l}\text { Pre-emptive } \\
=101(\text { MRD) }\end{array}$ & $\mathrm{CT}=100 \%$ & $1.7-7.4 \times 10^{7}$ & NA & $\mathrm{CR}=76 \%$ & $\begin{array}{c}\text { aGvHD=9\% } \\
\text { (grade } 3-4=4 \% \text { ) } \\
\text { cGvHD=59\% }\end{array}$ & $\begin{array}{c}\text { AML/MDS, } \\
\text { 3-yr DFS }=57 \% \\
3-y r \\
\text { NRM=7\% } \\
\text { ALL, 3-yr DFS }=49 \% \\
3-y r \\
\text { NRM }=11 \%\end{array}$ & $\begin{array}{l}\text { Post-DLI-GvHD } \\
\qquad \mathrm{ppx}=100 \%\end{array}$ \\
\hline
\end{tabular}


continued from the previous page

\begin{tabular}{|c|c|c|c|c|c|c|c|c|c|c|}
\hline $\begin{array}{l}\text { Study } \\
\text { T-cell depleted } \\
\text { haplo-HCT }\end{array}$ & $\begin{array}{l}\text { N of patients } \\
\text { (prospective/ } \\
\text { retrospective) }\end{array}$ & Diagnosis & $\begin{array}{l}\text { Indication } \\
\text { for DLI }\end{array}$ & $\begin{array}{l}\text { Treatments } \\
\text { before DLI }\end{array}$ & $\begin{array}{c}C^{3+} \\
\text { dose } / \mathrm{kg}\end{array}$ & $\begin{array}{l}\text { N. of DLI } \\
\text { (median) }\end{array}$ & $\begin{array}{l}\text { Disease } \\
\text { response }\end{array}$ & $\begin{array}{l}\text { Rate of } \\
\text { GvHDD }\end{array}$ & Survival & Notes \\
\hline $\begin{array}{l}\text { Yan et al. } \\
(2016)^{57 * \#}\end{array}$ & $\begin{array}{c}47 \\
\text { (haplo=31) } \\
\text { (prospective) }\end{array}$ & $\begin{array}{c}\mathrm{AML}=25 \\
\mathrm{ALL}=22\end{array}$ & $\begin{array}{c}\text { Pre-emptive }=47 \\
\text { (MRD) }\end{array}$ & $\mathrm{CT}=100 \%$ & $1.5-6.4 \times 10^{7}$ & $1-4$ & $\mathrm{NA}$ & $\begin{array}{c}\text { aGvHD }=19 \% \\
\text { (grade } 3-4=6 \%) \\
\text { cGvHD=79\% } \\
(\geq \text { moderate }=66 \%)\end{array}$ & $\begin{array}{c}\text { 1-yr DFS }=71 \% \\
1-\mathrm{yr} \\
\mathrm{NRM}=9 \% \\
1-\mathrm{yr} \\
\text { OS }=78 \%\end{array}$ & $\begin{array}{c}\text { Post-DLI-GvHD } \\
\text { ppx }=100 \%\end{array}$ \\
\hline $\begin{array}{l}\text { Yan et al. } \\
(2017)^{32 *}\end{array}$ & $\begin{array}{c}100 \\
\text { (haplo=62) } \\
\text { (prospective) }\end{array}$ & $\begin{array}{l}\mathrm{AML}=59 \\
\mathrm{ALL}=41\end{array}$ & Prophylactic $=100$ & $\mathrm{CT}=0 \%$ & $1.8-6.6 \times 10^{7}$ & NA & $\mathrm{NA}$ & $\begin{array}{c}\text { Haplo-HCT, } \\
\text { aGvHD =47\% } \\
\text { (grade } 3-4=10 \% \text { ) } \\
\text { cGvHD=63\% } \\
\text { ( } \geq \text { moderate=59\%) }\end{array}$ & $\begin{array}{c}\text { Haplo-HCT, } \\
3 \text {-yr DFS=51\% } \\
3 \text {-yr } \\
\text { NRM=18\% } \\
3-y r \\
\text { OS=49\% }\end{array}$ & $\begin{array}{c}\text { Post-DLI-GvHD } \\
\text { ppx }=100 \%\end{array}$ \\
\hline $\begin{array}{l}\text { Gao et al. } \\
(2018)^{54 *}\end{array}$ & $\begin{array}{c}31 \\
\text { (retrospective) }\end{array}$ & $\begin{array}{c}\text { AML }=21 \\
\text { ALL }=5 \\
\text { CML }=2 \\
\text { Other }=3\end{array}$ & Prophylactic $=31$ & $\mathrm{CT}=0 \%$ & $0.4-6.9 \times 10^{7}$ & 1 & NA & $\begin{array}{c}\text { aGvHD=58\% } \\
\text { (Grade 3-4=7\%) } \\
\text { cGvHD=39\% } \\
\text { (Mod-severe=29\%) }\end{array}$ & $\begin{array}{c}2 \text {-yr RFS }=32 \% \\
2-y r \text { NRM }=33 \% \\
2-y r \\
\text { OS }=40 \%\end{array}$ & $\begin{array}{c}\text { Post-DLI-GvHD } \\
\text { ppx }=100 \%\end{array}$ \\
\hline $\begin{array}{l}\text { Zeidan et al. } \\
(2014)^{28+}\end{array}$ & $\begin{array}{c}40 \\
\text { (retrospective) }\end{array}$ & $\begin{array}{c}\mathrm{AML}=16 \\
\mathrm{ALL}=3 \\
\mathrm{CML}=1 \\
\text { Lymphoma }=11 \\
\text { Other }=9\end{array}$ & $\begin{array}{c}\text { Therapeutic }=35 \\
\text { Pre-emptive }=5 \\
\text { (MRD+/MC) }\end{array}$ & $\begin{array}{c}\mathrm{CT} / \mathrm{RT}= \\
70 \%\end{array}$ & $\begin{array}{l}1 \times 10^{5} \\
\text { to } 1 \times 10^{8}\end{array}$ & $1-4(1)$ & $\begin{array}{c}\mathrm{CR}=30 \% \\
(\mathrm{MRD}=75 \%, \\
\text { Relapse }=26 \%)\end{array}$ & $\begin{array}{c}\text { aGvHD=25\% } \\
\text { (grade } 3-4=15 \% \text { ) } \\
\text { cGvHD }=8 \% \\
\text { (extensive=5\%) }\end{array}$ & $\begin{array}{l}\text { 8/12 responders } \\
\text { (67\%) alive, } \\
\text { in CR at } \\
17.5 \text { months }\end{array}$ & \\
\hline
\end{tabular}

\begin{tabular}{|c|c|c|c|c|c|c|c|c|c|c|}
\hline $\begin{array}{l}\text { Ghiso et al. } \\
(2015)^{29+}\end{array}$ & $\begin{array}{c}42 \\
\text { (retrospective) }\end{array}$ & $\begin{array}{c}\mathrm{AML}=22 \\
\mathrm{ALL}=9 \\
\mathrm{HL}=10 \\
\mathrm{MM}=1\end{array}$ & $\begin{array}{c}\text { Therapeutic }=22 \\
\text { Pre-emptive }=20 \\
\text { (MRD) }\end{array}$ & $\begin{array}{c}\mathrm{CT} / \mathrm{RT}= \\
100 \%\end{array}$ & $\begin{array}{l}1 \times 10^{3} \\
\text { to } 1 \times 10^{7}\end{array}$ & $\begin{array}{c}1-6 \\
(\text { mean-2.5) }\end{array}$ & $\begin{array}{l}\mathrm{CR}=36 \% \\
\mathrm{CR} \text { in } \mathrm{MRD} \\
\quad=45 \%\end{array}$ & $\begin{array}{l}\text { aGvHD }=33 \% \\
\text { (grade } 3-4=5 \% \text { ) } \\
\text { cGvHD }=0 \%\end{array}$ & $\begin{array}{l}\text { Leukemia, median } \\
\text { response }=4 \mathrm{~m}(2-10) \\
\text { median survival } \\
\quad=7 \mathrm{~m}(1-15) \\
\text { LL, median response } \\
=9 \mathrm{~m}(3-28), \\
\text { median survival } \\
=18 \mathrm{~m}(4-34)\end{array}$ & \\
\hline $\begin{array}{l}\text { Goldsmith et al. } \\
(2017)^{30+}\end{array}$ & $\begin{array}{c}21 \\
\text { (retrospective) }\end{array}$ & $\begin{array}{c}\text { AML/MDS }=1 \\
\text { ALL }=2 \\
\text { CML }=2 \\
\text { Lymphoma }= \\
\text { Other }=9\end{array}$ & $\begin{array}{l}6 \text { Therapeutic }=19 \\
\text { Pre-emptive }=2(\mathrm{MC}) \\
1\end{array}$ & $\mathrm{CT}=76 \%$ & $0.01-3 \times 10^{7}$ & $1-5$ & $\mathrm{CR}=32 \%$ & $\begin{array}{c}\text { aGvHD }=33 \% \\
\text { (grade } 3-4=5 \% \text { ) } \\
\text { cGvHD }=26 \% \\
\text { (extensive }=0 \% \text { ) }\end{array}$ & $\begin{array}{c}\text { Hematologic } \\
\text { relapse, } \\
\text { 4-m RFS=8\% } \\
\text { 4-m OS=29\% } \\
\text { EM relapse, } \\
\text { 4-m RFS=43\% } \\
\text { 4-m OS=71\% }\end{array}$ & PBSC haplo-HCT \\
\hline $\begin{array}{l}\text { Cauchois et al. } \\
(2018)^{67+}\end{array}$ & $\begin{array}{c}36 \\
\text { (retrospective) }\end{array}$ & \multicolumn{2}{|c|}{$\begin{array}{l}\text { AML/MDS }=25 \text { Prophylactic }=36 \\
\text { ALL }=2 \\
\text { Lymphoma }=6 \\
\text { Other }=3\end{array}$} & $\mathrm{CT}=0 \%$ & $0.1-2.5 \times 10^{6}$ & $1-3$ & NA & \multicolumn{2}{|c|}{$\begin{array}{cc}1 \text {-yr CI of mod-severe } 1-\mathrm{yr} P F S=83 \% \\
\mathrm{GvHD}=33 \% & 1-\mathrm{yr} \mathrm{NRM}=9 \% \\
1-\mathrm{yr} & \text { OS }=76 \%\end{array}$} & PBSC graft $=31$ \\
\hline
\end{tabular}

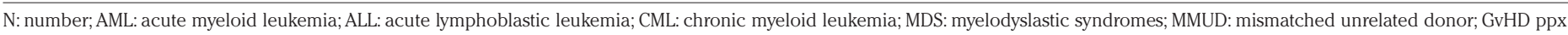

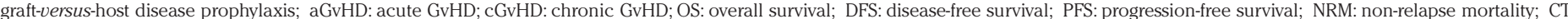

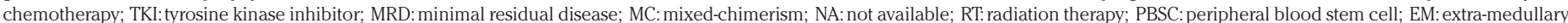

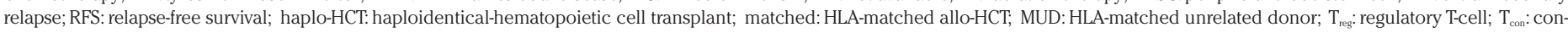

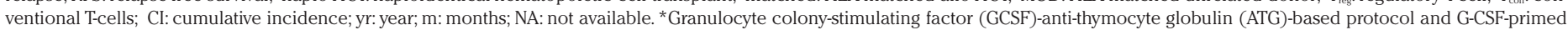

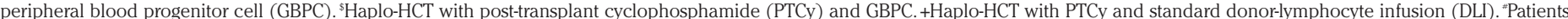

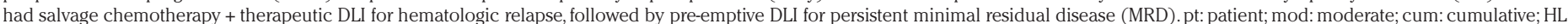
Hodgkin lymphoma. 


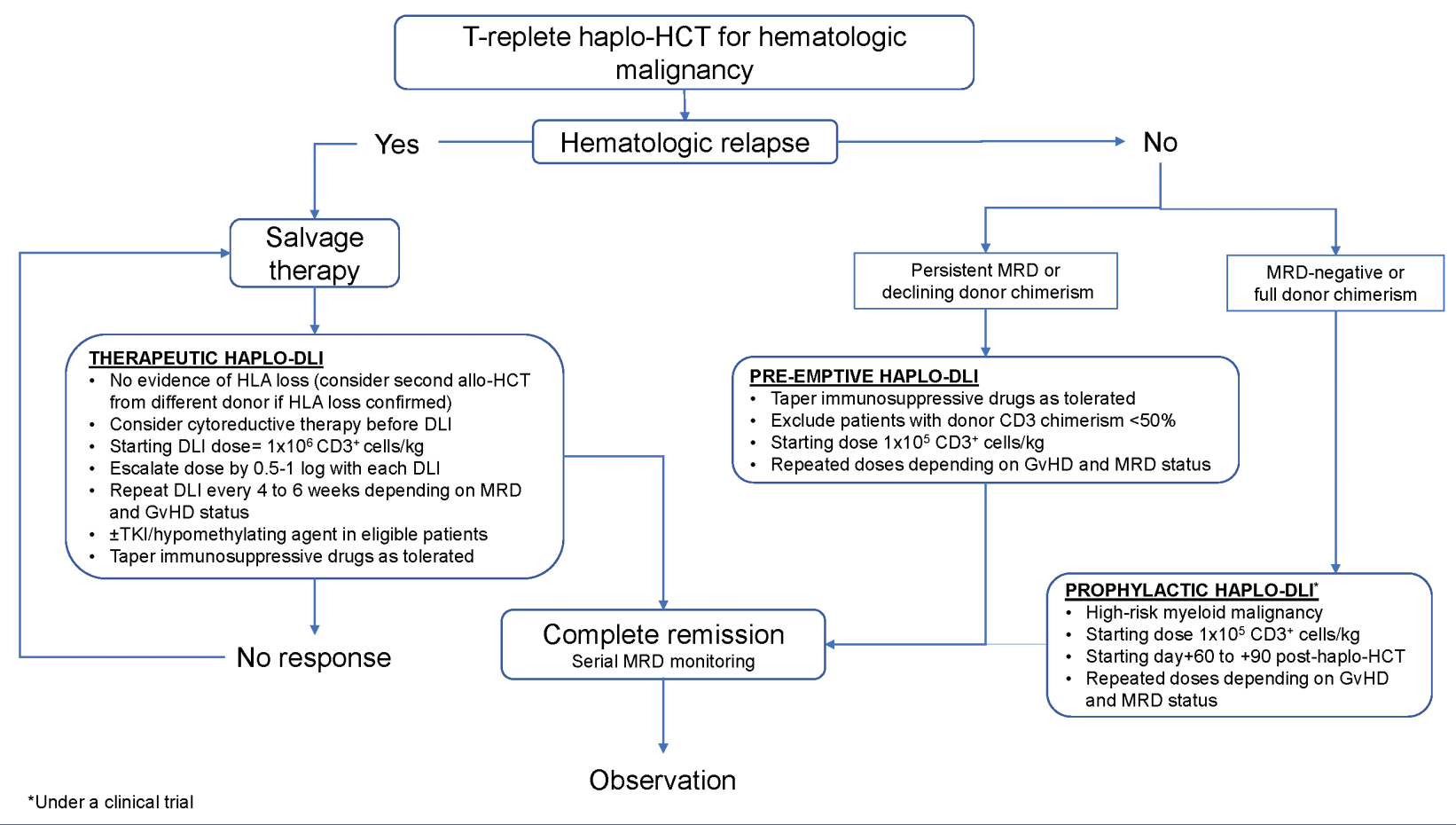

Figure 1. Proposed treatment algorithm of therapeutic, pre-emptive and prophylactic donor-lymphocyte infusion (DLI) following T-cell replete haploidentical hematopoietic cell transplantation (HCT). HLA: human leukocyte antigen; MRD: minimal residual disease; GvHD: graft-versus-host disease; TKI: tyrosine kinase inhibitor.

specific MRD management strategies. DLI should not be used in patients who have converted to host chimerism due to increased risk of marrow aplasia. ${ }^{49}$ An alternative strategy for such patients would be to undergo a second allo-HCT from the same or from a different donor. It is important to weigh the risk of GvHD and marrow aplasia versus the potential benefit of reducing the disease relapse when considering pre-emptive DLI for MRD or MC.

\section{Prophylactic haplo-donor-lymphocyte infusion}

Prophylactic DLI from a matched donor has been studied in patients with high-risk myeloid malignancies and was associated with improved disease-specific outcomes and low NRM. ${ }^{11,50,51}$ It can contribute to immune reconstitution and reduce the risk of infection, ${ }^{52}$ which is a major challenge after a T-cell depleted haplo-HCT. A matchedpair analysis by the EBMT showed improved OS in highrisk AML recipients who received prophylactic DLI from a matched donor $(70 \%$ vs. $40 \%$ in controls; $P=0.027) .{ }^{53}$ Inclusion criteria differ among published studies in their definition of high-risk disease. Most reports included patients with primary induction failure acute leukemia, high disease risk index, active disease before allo-HCT or the presence of high-risk mutations (i.e. TP53, ASXL1, RUNX1) in myeloid malignancies. ${ }^{54.57}$ One of the first experiences with prophylactic haplo-DLI was in the setting of autologous-HCT. Nagler et al. reported outcomes of 26 patients who received multiple haplo-DLI (with/without IL-2) after an autologous-HCT. This approach was feasible in inducing GvHD, but higher cell doses led to increased toxicity. ${ }^{58}$ The timing of prophylactic-DLI is also important as decreasing the interval between allo-HCT and DLI will likely increase the risk of aGvHD. ${ }^{13}$ The activity of ATG, given as a part of conditioning, may persist for weeks, and residual ATG may negatively impact prophylactically infused donor lymphocytes ${ }^{59}$ At the same time, the administration of haploDLI as early as $d+45$ was feasible in single center studies. ${ }^{32,56}$ It may be reasonable to administer prophylactic haplo-DLI before $\mathrm{d}+90$ given that median time to relapse after allo-HCT is approximately three months.

\section{Prophylactic donor-lymphocyte infusion in T-cell depleted haplo-hematopoietic cell transplantation}

Early experience with prophylactic haplo-DLI was with T-cell-depleted haplo-HCT where donor lymphocytes were infused after a CD $34^{+}$cell-selected graft to enhance immune reconstitution. ${ }^{60-62}$ Perruccio et al. showed that infusion of donor-derived non-alloreactive $\mathrm{T}$ cells specific for cytomegalovirus (CMV) and aspergillus resulted in the rapid development of $\mathrm{T}$-cell responses against these pathogens without inducing GvHD. ${ }^{63}$ Another prospective study utilized $\mathrm{CD} 8^{+}$T-cell depleted DLI, which resulted in aGvHD in $26 \%$ of patients with 2 -year PFS of $45 \% .{ }^{62}$ Donor-derived $T$ regulatory cells $\left(\mathrm{T}_{\text {regs }}\right)$, co-infused with conventional $T$ cells $\left(T_{\text {cons }}\right)$ were shown to protect recipients against GvHD. ${ }^{64}$ In a prospective study, patients received $T_{\text {regs }}(d-4)$ followed by a megadose of CD34 cells and $\mathrm{T}_{\text {cons }}$ on $\mathrm{d} 0$ from a haploidentical donor without any post-transplant immunosuppression. Only $15 \%$ of the patients developed $\geq$ grade $2 \mathrm{aGvHD}$ and DFS was $56 \%$ at 18 months. ${ }^{61}$ In another prospective study by Gilman et al., 34 pediatric patients were infused an unmanipulated prophylactic haplo-DLI with MTX between $\mathrm{d}+30$ and $\mathrm{d}+42$ after a T-cell depleted/CD34+ haplo-HCT. The intervention was safe and 2-year NRM 
and OS were $25 \%$ and $63 \%$, respectively. ${ }^{60}$ Lewalle et al. reported the outcomes of 12 patients who received prophylactic haplo-DLI starting on $\mathrm{d}+28$ after $\mathrm{T}-/ \mathrm{B}-$-cell depleted haplo-HCT in a prospective study. One-year RFS, NRM, and OS were $50 \%, 0 \%$, and $50 \%$, respectively. ${ }^{65}$ Despite the encouraging results with prophylactic infusion of $\mathrm{T}$-cell subset after a T-cell deplete/CD34+ selected haplo-HCT, its widespread adoption has been challenging as cell selection remains a labor-intensive and expensive process.

\section{Prophylactic donor-lymphocyte infusion in T-cell replete} haplo-hematopoietic cell transplantation

The Chinese group has led the way by incorporating prophylactic GBPC in high-risk malignancies after a haplo-HCT with the GCSF-ATG-based protocol. In a retrospective study by Wang et al., ${ }^{61}$ patients with high-risk leukemia who underwent GBPC infusion were compared to 27 patients who received routine care after an haploHCT. Prophylactic GBPC was associated with lower relapse rate ( $36 \%$ vs. $55 \% ; P=0.017)$ and superior estimated 3 -year survival (31\% vs. $11 \% ; P=0.001)$ compared to routine care. There was no difference in NRM between the two groups. ${ }^{6}$ A prospective study by the same group enrolled 62 patients with high-risk acute leukemia. All patients received prophylactic GPBC between $d+45$ and $\mathrm{d}+60$ and further DLI were guided by MRD and GvHD status. Three-year DFS, NRM, and OS were 51\%, 18\%, and $49 \%$, respectively. Acute and chronic GvHD were seen in $47 \%$ and $63 \%$ patients, respectively. Outcomes were similar between recipients of haploidentical $(n=62)$ versus matched donor $(\mathrm{n}=38)$ prophylactic DLI. ${ }^{32}$

Jaiswal et al. reported their prospective experience with prophylactic GBPC in the T-cell replete haplo-HCT/PTCy setting. Twenty-one patients with AML (not in remission) received up to three doses of haplo-GPBC $(d+21, d+35$ and $d+60)$. They were compared with 20 patients who received routine monitoring after haplo-HCT. At 18 months, CIR, PFS, and OS were $21 \%$ versus $66 \%$; $62 \%$ versus $25 \%$ and $71 \%$ versus $35 \%$ in DLI and routine care cohorts, respectively. Incidence of aGvHD was $31 \%$, while incidence of chronic GvHD was $41 \%$ after GBPC infusions. NRM was equivalent between the groups. ${ }^{56}$ Recently, Cauchios et al. reported outcomes of 36 patients who received prophylactic haplo-DLI after a haploHCT/PTCy. One-year PFS and OS were $76 \%$ and $83 \%$, respectively. The cumulative incidence of relapse was $16 \%$ and the incidence of DLI-associated GvHD was $33 \% .^{67}$

\section{Practical aspects of haplo-donor-lymphocyte infusion}

\section{Cell dose}

The $\mathrm{CD}^{+}$T-cell dose ranged from 0.01 to $8.8 \times 10^{8}$ mononuclear cells/kg in reports on therapeutic DLI from a matched donor. ${ }^{7}$ A study reported a relatively lower rate of GvHD with an escalating cell dose regimen versus a single bulk infusion of DLI from HLA-matched donors. Disease responses were similar between the two approaches. ${ }^{68}$ There was no dose-response relationship with GvHD or disease response rates in haplo-DLI in the setting of T-cell depleted haplo-HCT. ${ }^{28,29}$ The average starting dose for therapeutic haplo-DLI in the T-cell replete haplo-HCT/PTCy setting was 1 or 2 log lower than the standard DLI dose $\left(1 \times 10^{7} \mathrm{CD}^{+}\right.$cells $\left./ \mathrm{kg}\right)$ from HLA-matched donors. In a report on 40 patients, a cell dose of $1 \times 10^{6} \mathrm{CD}^{+}$cells $/ \mathrm{kg}$ was associated with grade 2 $4 \mathrm{aGvHD}$ in $17 \%$ of patients, and a CR rate of $27 \% .{ }^{28}$ Goldsmith et al. used the same dose in 21 patients; only seven $(33 \%$ ) developed aGvHD (grade $3-4$ aGvHD in 1 patient). ${ }^{30}$ These incidences of a GvHD were lower than those reported by the Chinese group using haplo-GBPC at 1 to 2 log higher cell dose with the GCSF-ATG-based protocol. A starting cell dose of $1 \times 10^{7}$ to $1 \times 10^{8} \mathrm{CD}^{+}$ cells/kg was associated with grade $2-4$ aGvHD in 50-60\% (grade 3-4 aGvHD approximately $30 \%$ ) of patients. ${ }^{31,38,69}$ Subsequent reports by Yan et al. showed a reduced incidence of aGvHD with the routine use of short-term GvHD prophylaxis after GBPC infusion. ${ }^{39}$ Available data suggest that $1 \times 10^{6} \mathrm{CD}^{+}$cells $/ \mathrm{kg}$ is a reasonable starting dose with appropriate repeated dose escalation every 4-6 weeks based on disease response and GvHD for therapeutic haplo-DLI in T-replete haplo-HCT with PTCy. Clinical trials are needed to establish the optimal timing and cell dose in prophylactic and T-cell depleted haploHCT settings. Published studies have used wide-ranging repeated non-escalating cell doses for pre-emptive or prophylactic DLI. . $^{32,37,54}$

\section{The end point of donor-lymphocyte infusion therapy}

It is important to establish the goal of DLI therapy beforehand as each DLI is associated with increased risk of GvHD. Patients with DLI-responsive relapse usually respond within 2-3 months. ${ }^{7}$ Repeated infusions of escalating doses of therapeutic DLI can be administered until $\mathrm{CR}$ is achieved (ideally an MRD-negative status) or the patient develops clinically significant GvHD. Patients should be evaluated for GvHD, donor chimerism and disease response after each DLI. Pre-emptive DLI for MRD persistence after allo-HCT may be stopped once the achievement of MRD negativity, significant GvHD or a hematologic relapse occurs. Donor chimerism should be assessed after each pre-emptive DLI for MC. Pre-emptive DLI may be stopped once $\geq 90 \%$ donor chimerism is achieved. As noted above, DLI can result in marrow aplasia in those patients who have converted to host chimerism. There is no standard duration of prophylactic DLI outside a clinical trial. In these circumstances, each dose of prophylactic haplo-DLI should be used with caution, balancing the risk of disease relapse and GvHD.

\section{Traditional donor-lymphocyte infusion versus granulocyte colony-stimulating factor-primed peripheral blood progenitor cell infusion}

Standard DLI uses freshly collected unmanipulated donor lymphocytes. This approach privileges tumor alloreactivity over the risk of GvHD. GCSF promotes Tcell hypo-responsiveness in marrow grafts by increasing the number of plasmacytoid dendritic cells and monocytes. It also reduces the expression of co-stimulatory CD28/B7 on monocytes, B and $\mathrm{T}$ cells, ${ }^{70}$ promotes macrophage ${ }^{71}$ and $T$-cell polarization in the BM graft towards the more tolerogenic pattern. This property is maintained even after in vitro mixture of G-CSF primed $\mathrm{BM}$ and PBSC grafts. $^{72,73}$ The Chinese group has reported their extensive experience with using GBPC instead of unmanipulated DLI. Huang et al. reported the outcomes of 20 patients who received therapeutic GBPC from hap- 
loidentical donors (the majority of whom received salvage therapy prior to GBPC infusion); CR was achieved in $75 \%$ of patients and the rates of acute and chronic GvHD were $55 \%$ and $64 \%$, respectively. ${ }^{38}$ In another study of pre-emptive GBPC infusion for MRD after an allo-HCT (haploidentical related donor: $n=29$; matchedrelated donor: $\mathrm{n}=26$ ), the incidences of acute and chronic GvHD were $31 \%$ and $43 \%$, respectively. Routine debulking chemotherapy and short-term immunosuppression were used in most studies using GBPC. ${ }^{31} \mathrm{~A}$ prospective observational study by Jaiswal et al. used prophylactic-GBPC in the setting of T-cell replete haploHCT/PTCy. ${ }^{56}$ The incidence of aGvHD was comparable but $\mathrm{cGvHD}$ was higher than that reported with unmanipulated haplo-DLI in the PTCy setting. ${ }^{28-30}$ A recent report from Mexico showed that administration of GCSF-primed whole blood units (median cell count $6.7 \times 10^{6} \mathrm{CD}^{+}$cells $/ \mathrm{kg}$ ) from haploidentical donors is safe, with disease responses and improvement in $\mathrm{MC}$ in a subset of patients. ${ }^{74}$ Whole blood units can potentially reduce the cost associated with haplo-DLI in developing countries. Long-term immune tolerance after PTCy may be enough to overcome the immunological barrier of haplo-DLI, and GCSF priming may not be required in this setting. Comparative studies between unmanipulated DLI versus GBPC in the setting of haplo-HCT/PTCy are needed.

\section{Role of concurrent immunosuppression}

Graft-versus-host disease is the main limiting toxicity of DLI, and short-term immunosuppression with DLI may improve the safety of DLI. Yan et al. reported aGvHD in $31 \%$ of patients after pre-emptive GBPC infusion for $\mathrm{MRD}$ persistence after $\mathrm{T}$-cell replete haplo-HCT. ${ }^{31}$ All patients received CSA or low-dose MTX for 6-8 weeks after GBPC. There was no difference in acute and chronic GvHD rates between CSA and MTX. MTX was associated with lower relapse rate (38\% vs. $81 \% ; P=0.029)$ and better DFS (52\% vs. 16\%; $P=0.06)$. Patients who received MTX had higher absolute lymphocyte count compared to those who received CSA, which may have contributed to better GvT effect. ${ }^{39}$ The same group also showed that patients receiving GvHD prophylaxis for 6-8 weeks had a lower cumulative incidence of grade 3-4 aGvHD than patients receiving prophylaxis for 4-6, 2-4, and $<2$ weeks $(9 \%, 14 \%, 32 \%$, and $50 \%$, respectively; $P=0.018) .{ }^{69}$ In a retrospective study, Mo et al. used pre-emptive chemoDLI for MRD persistence along with routine prophylaxis with CSA or MTX (haploidentical donor 6-8 weeks; matched donor 4-6 weeks). The incidence of a GvHD was only 9\% (grade 3-4 aGvHD: 4\%) in their cohort of 101 patients. ${ }^{36}$ It is important to note here that haplo-DLI without concurrent immunosuppression in the T-cell replete haplo-HCT/PTCy setting has been reported to have a similar incidence of GvHD compared to the GCSFATG-based haplo-HCT protocol, which routinely uses prophylactic immunosuppression with DLI. ${ }^{28}$ The potential impairment of the DLI-mediated GvT effect by CSA or MTX is a concern when managing a hematologic relapse. It is reasonable to add short-term MTX after a pre-emptive or prophylactic haplo-DLI, especially in patients with a history of GvHD. ${ }^{56}$ There are no data available on concurrent immunosuppression with therapeutic haplo-DLI in the T-cell replete haplo-HCT/PTCy setting.

\section{Combination of systemic therapies with donor-lymphocyte infusion}

Administration of salvage therapy before the infusion of DLI may improve its efficacy by reducing the tumor burden and supporting in vivo expansion of infused $T$ cells. In this regard, chemotherapy helps eliminate regulatory donor $\mathrm{T}$ cells and create a favorable immunological environment for DLI by increasing serum levels of IL-7 that favors peripheral expansion of $\mathrm{T}$ cells. ${ }^{75}$ In the retrospective study by Zeidan et al., patients who received a cytoreductive therapy had better CR rates compared to those who received unmanipulated haplo-DLI without any preceding therapy (39\% vs. $8 \%){ }^{28}$ This beneficial effect of pre-DLI chemotherapy was not seen in a similar report by Goldsmith et al. ${ }^{30}$

The downside of pre-DLI chemotherapy is tissue injury and inflammatory cytokine surge which may increase the risk of GvHD, especially when used closer to the allo$\mathrm{HCT}^{76}$ Intensive chemotherapy after an allo-HCT is poorly tolerated, and infectious complications are common. . $^{4,76}$ Recently, hypomethylating agents (i.e. azacitidine, decitabine) have been used with DLI for relapsed AML/MDS. Azacitidine can induce allogeneic $\mathrm{CD}^{+} \mathrm{T}$ cell response by enhancing the expression of epigenetically silenced tumor-associated antigens. ${ }^{77}$ A combination of a hypomethylating agent and DLI is safe with no apparent increase in GvHD or infection risk compared to DLIalone. ${ }^{78,79}$ In a prospective study using azacitidine with DLI for relapsed disease after HLA-matched allo-HCT, the CR rate was $23 \%$ and the 2 -year OS was $17 \% .^{80}$ Another retrospective study utilizing decitabine followed by DLI for relapsed myeloid malignancies showed an overall response rate of $25 \%$ with 2 -year OS of $11 \% .^{81}$ Drugs targeting specific molecular anomalies (BCR-ABL1, FLT3-ITD, IDH1, IDH2) are increasingly being incorporated in the treatment of disease relapse or as maintenance therapy after allo-HCT. ${ }^{82,83}$ These drugs are safer compared to traditional salvage chemotherapy and may provide benefit when administered in combination with DLI. ${ }^{84}$

\section{Immune escape after haplo-hematopoietic cell transplantation}

Recent data have shed light on mechanisms of immune escape causing disease relapse after haplo-HCT. In haploSCT, HLA haplotype mismatched in the donor/recipient pair was replaced by a shared parent haplotype (uniparental disomy) in 5 of 17 patients with relapsed AML post-haplo-HCT. ${ }^{85}$ In a subsequent retrospective analysis of 69 patients who relapsed after haplo-HCT, mismatched-HLA haplotype loss accounted for $33 \%$ of the relapses. ${ }^{86}$ Based on retrospective studies, a second alloHCT using a donor with a different mismatched haplotype or a mismatched unrelated donor may induce a better GvT effect compared to same donor from the first haplo-HCT. ${ }^{86,87}$ At present, there is no standardized method of detecting loss of mismatched HLA haplotype in leukemic cells. HLA-allele specific quantitative polymerase chain reaction is required to quantify recipientand donor-specific alleles to confirm uniparental disomy in low-burden disease relapse. .6,88 $^{86}$

Historically speaking, most patients receiving therapeutic DLI relapse and succumb to their disease. Close monitoring of MRD and donor chimerism after a successful therapeutic haplo-DLI is important to identify the 
patients who are at high-risk of subsequent relapses. Mo et al. reported that patients with persistent MRD after DLI had increased relapse risk $(P=0.001)$, resulting in poor DFS $(P=0.004){ }^{36}$ In a prospective study of 47 patients $(66 \%$ received haplo-HCT), $M R D$-guided repeated administration of pre-emptive chemo-DLI was effective in reducing the risk of subsequent relapse after achieving initial disease response. The one-year CIR, DFS, and OS were $22 \%, 71 \%$, and $78 \%$, respectively (Figure 1). ${ }^{57}$

\section{Future directions}

\section{Donor-derived natural killer cells}

Natural killer cells may play a role in tumor alloreactivity in the setting of mismatched or haploidentical transplant. A recent study showed a marked reduction in donor-derived NK cells in the recipients of PTCy, leading to blunting of NK-cell alloreactivity. ${ }^{89}$ In a pilot study, prophylactic infusion of $\mathrm{CD}^{+} 6^{+} / \mathrm{CD}^{+}$cells after haploHCT/PTCy in patients with refractory active disease was safe and associated with rapid immune reconstitution. ${ }^{55}$ The same group used prophylactic DLI primed with abatacept (CTLA4Ig), which selectively suppresses T-cell alloreactivity without interfering with NK-cell activation. Abatacept with DLI was associated with reduced incidence of aGvHD (10\% vs. $31 \%$ ) and improved relapsefree survival compared to prophylactic DLI alone..$^{90}$ In a phase I study by Ciurea et al., donor-derived NK cells expanded ex vivo were infused prophylactically before and after haplo-HCT in high-risk myeloid malignancies. The intervention was safe and associated with improved NK-cell number and function, lower viral infections, and low relapse rate when compared to a historical control group..$^{91}$ Several methods to enhance NK-cell alloreactivity, including combination with immunomodulatory drugs, ${ }^{92}$ use of cytokine-activated NK cells, ${ }^{93}$ and selection of alloreactive single $\mathrm{KIR}^{+} \mathrm{NK}$ cells,${ }^{94}$ are under investigation.

\section{Engineered donor-lymphocyte infusion}

Different strategies are being explored to modify DLI composition and reduce the risk of GvHD while maintaining antitumor activity. ATIR $101^{\circ}$ is a haplo-DLI product with alloreactive $\mathrm{T}$ cells depleted by ex vivo photodepletion. ${ }^{20}$ In a pooled analysis of two prospective trials, 37 patients received prophylactic ATIR101 ${ }^{\circ}$ after T-cell depleted haplo-HCT. One-year relapse rate, NRM and OS were $8 \%, 33 \%$ and $58 \%$, respectively. Interestingly, aGvHD (grade 3-4) and severe cGvHD were seen in 5\% and $0 \%$ of the patients, respectively. ${ }^{95}$ Alloanergized DLI generated ex vivo was infused on $\mathrm{d}+35$ after a CD34+ selected haplo-HCT in a phase I study. These donor lymphocytes with the reduced donor-specific alloreactivity expanded in vivo and contributed to immune reconstitution. ${ }^{96}$ Another strategy is to insert an inducible suicide gene in donor lymphocytes so that they can be selectively eliminated to treat DLI-associated GvHD. ${ }^{21,97}$ A recent analysis on 100 children with acute leukemia given a titrated number of donor $\mathrm{T}$ cells transduced with the inducible caspase-9 safety switch after haplo-HSCT showed an $82 \%$ probability of relapse-free survival. ${ }^{98}$

Chimeric antigen receptor T-cell (CAR-T) therapy has emerged as a potent form of adoptive cellular therapy. Two CD19 CAR-T-cell therapies have been approved by the US Food and Drug Administration (FDA) and the European Medicines Agency (EMA) for relapsed/refractory high-grade B-cell lymphoma and B-ALL. ${ }^{99,100}$ Prophylactic infusion of CD19 CAR-T cells from a haploidentical donor was found to be safe with only mild aGvHD in one report. ${ }^{101}$ There are reports of therapeutic or pre-emptive donor-derived CAR-T-cell infusion with a small number of patients achieving durable remissions. CAR-T-cell-associated GvHD appears to be rare and of mild severity. ${ }^{102}$ Selective depletion of $\mathrm{CD}^{+} \alpha \beta-\mathrm{TCR}^{+} \mathrm{T}$ cells (thought to be the principal mediators of GvHD) to enrich DLI with $\mathrm{CD}^{+} \gamma \delta-\mathrm{TCR}^{+} \mathrm{T}$ cells and $\mathrm{CD}^{-} \mathrm{CD}^{-} 6^{+}$ $\mathrm{NK}$ cells is also an attractive strategy to reduce the risk of GvHD while maintaining tumor alloreactivity. ${ }^{103}$ Maschan et al. infused low-dose $\left(1 \times 10^{5} \mathrm{CD}^{+}\right.$cells $\left./ \mathrm{kg}\right)$ CD45RA-depleted DLI (memory $\mathrm{T}$ cell) in 25 patients after TCR $\alpha / \beta$-depleted haplo-HCT. The intervention was safe and associated with the expansion of cytomegalovirus-specific T cells in the recipients. ${ }^{52}$

\section{Donor-lymphocyte infusion with immunomodulatory drugs}

Immunomodulation with checkpoint inhibitors and targeted agents may enhance the efficacy of DLI. This may allow lower $\mathrm{CD}^{+}$cell dose while maintaining tumor alloreactivity. Blinatumomab (a CD19-CD3 bispecific Tcell engager) has been used with DLI for relapsed B-ALL. In a recent report of 14 patients, it appears to be safe with high response rates. ${ }^{104}$ In a prospective phase II study, DLI was administered with azacitidine and lenalidomide in patients with molecular or hematologic relapse of myeloid malignancies. The combination was relatively safe and the CR rates were $67 \%$ in MRD and $43 \%$ in hematologic relapse. ${ }^{80}$ Interferon- $\gamma$ (IFN $\gamma$ ) induced reexpression of epigenetically silenced MHC class II antigens in relapsed AML clones after allo-HCT. ${ }^{105}$ One could hypothesize that treating a patient with IFN $\gamma$ before haplo-DLI may result in better tumor alloreactivity, although it may also increase the risk of GvHD.

\section{Conclusions}

- Unmanipulated DLI from a haploidentical donor appears to be relatively safe and reasonably effective in patients who relapse after a T-cell replete haplo-HCT. Patients given haplo-DLI should be enrolled in a clinical trial whenever possible, as data regarding optimal cell dose, timing and role of concurrent systemic therapies with haplo-DLI are limited. Information about the application of unmanipulated DLI after T-cell depleted transplantation is limited, which is why dosing should be managed with caution.

- The risk of GvHD after unmanipulated DLI in the haplo-HCT/PTCy setting is comparable to an unmanipulated DLI from an HLA-matched donor.

- Cytoreductive therapy prior to DLI from a haploidentical donor should be considered in patients with a hematologic relapse after haplo-HCT.

- Pre-emptive haplo-DLI may play a role in reducing disease relapse in patients with persistent MRD or mixeddonor chimerism after haplo-HCT; however, more studies are needed.

- Patients with high-risk myeloid malignancies may benefit from a prophylactic haplo-DLI, which should ide- 
ally be used in the setting of a clinical trial.

- The administration of manipulated DLI after T-cell depleted or T-cell replete haploidentical transplantation, such as allodepleted or gene-modified T cells, should only be performed in the setting of a clinical trial.

- Patients should be monitored closely with frequent disease-specific MRD testing and donor-chimerism after DLI administration.

- Mismatched-HLA allele loss was detected in one- third of leukemia relapses after a haplo-HCT. Such patients are unlikely to benefit from DLI from the original donor. A second allo-HCT from a related donor with a different mismatched haplotype or a mismatched unrelated donor may be considered if HLA-loss is confirmed.

\section{Acknowledgments}

The authors would like to thank Vidya B. Pai, MD, for assistance with proofreading.

\section{References}

1. Bejanyan N, Weisdorf DJ, Logan BR, et al. Survival of patients with acute myeloid leukemia relapsing after allogeneic hematopoietic cell transplantation: a center for international blood and marrow transplant research study. Biol Blood Marrow Transplant. 2015;21(3):454-459.

2. D'Souza A, Fretham C. Current Uses and Outcomes of Hematopoietic Cell Transplantation (HCT): CIBMTR Summary Slides, 2018.

3. Ciurea SO, Labopin M, Socie G, et al. Relapse and survival after transplantation for complex karyotype acute myeloid leukemia: A report from the Acute Leukemia Working Party of the European Society for Blood and Marrow Transplantation and the University of Texas MD Anderson Cancer Center. Cancer. 2018;124(10):2134-2141.

4. Kolb HJ, Mittermuller J, Clemm C, et al. Donor leukocyte transfusions for treatment of recurrent chronic myelogenous leukemia in marrow transplant patients. Blood. 1990;76(12):2462-2465

5. Pati AR, Godder K, Lamb L, et al. Immunotherapy with donor leukocyte infusions for patients with relapsed acute myeloid leukemia following partially mismatched related donor bone marrow transplantation. Bone Marrow Transplant. 1995; 15(6):979-981.

6. Sica S, Di Mario A, Salutari P, et al. Chemotherapy and recombinant human granulocyte colony-stimulating factor primed donor leukocyte infusion for treatment of relapse after allogeneic bone marrow transplantation. Bone Marrow Transplant. 1995;16(3):483-485.

7. Deol A, Lum LG. Role of donor lymphocyte infusions in relapsed hematological malignancies after stem cell transplantation revisited. Cancer Treat Rev. 2010;36(7):528-538.

8. Chalandon Y, Passweg JR, Schmid C, et al. Outcome of patients developing GVHD after DLI given to treat CML relapse: a study by the Chronic Leukemia Working Party of the EBMT. Bone Marrow Transplant. 2010;45(3):558-564.

9. Takami A, Yano S, Yokoyama H, et al. Donor lymphocyte infusion for the treatment of relapsed acute myeloid leukemia after allogeneic hematopoietic stem cell transplantation: a retrospective analysis by the Adult Acute Myeloid Leukemia Working Group of the Japan Society for Hematopoietic Cell Transplantation. Biol Blood Marrow Transplant. 2014;20(11): 1785-1790.

10. Schmid C, Labopin M, Nagler A, et al. Donor lymphocyte infusion in the treatment of first hematological relapse after allogeneic stem-cell transplantation in adults with acute myeloid leukemia: a retrospective risk factors analysis and comparison with other strategies by the EBMT Acute Leukemia Working Party. J Clin Oncol. 2007;25(31): 4938-4945.

11. Jedlickova Z, Schmid C, Koenecke C, et al. Long-term results of adjuvant donor lymphocyte transfusion in AML after allogeneic stem cell transplantation. Bone Marrow Transplant. 2016;51(5):663-667.

12. Haines HL, Bleesing JJ, Davies SM, et al. Outcomes of donor lymphocyte infusion for treatment of mixed donor chimerism after a reduced-intensity preparative regimen for pediatric patients with nonmalignant diseases. Biol Blood Marrow Transplant. 2015;21(2):288-292

13. Liga M, Triantafyllou E, Tiniakou M, et al. High alloreactivity of low-dose prophylactic donor lymphocyte infusion in patients with acute leukemia undergoing allogeneic hematopoietic cell transplantation with an alemtuzumab-containing conditioning regimen. Biol Blood Marrow Transplant. 2013;19(1):75-81

14. Passweg JR, Baldomero $\mathrm{H}$, Bader $\mathrm{P}$, et al. Hematopoietic stem cell transplantation in Europe 2014: more than 40000 transplants annually. Bone Marrow Transplant. 2016:51(6):786-792

15. Kanakry CG, Fuchs EJ, Luznik L. Modern approaches to HLA-haploidentical blood or marrow transplantation. Nat Rev Clin Oncol. 2016;13(1):10-24.

16. Huang XJ, Liu DH, Liu KY, et al. Haploidentical hematopoietic stem cell transplantation without in vitro $\mathrm{T}$-cell depletion for the treatment of hematological malignancies. Bone Marrow Transplant. 2006;38(4):291-297.

17. Ciceri F, Labopin M, Aversa F, et al. A survey of fully haploidentical hematopoietic stem cell transplantation in adults with high-risk acute leukemia: a risk factor analysis of outcomes for patients in remission at transplantation. Blood. 2008;112(9):3574-3581.

18. Locatelli F, Merli P, Pagliara D, et al. Outcome of children with acute leukemia given HLA-haploidentical HSCT after alphabeta T-cell and B-cell depletion. Blood. 2017;130(5):677-685

19. Lang PJ, Schlegel PG, Meisel R, et al. Safety and Efficacy of Tcralpha/Beta and CD19 Depleted Haploidentical Stem Cell Transplantation Following Reduced Intensity Conditioning in Children: Results of a Prospective Multicenter Phase I/II Clinical Trial. Blood. 2017;130(Suppl 1):214.

20. Roy DC, Lachance S, Cohen S, et al. Allodepleted T-cell immunotherapy after haploidentical haematopoietic stem cell transplantation without severe acute graft- versus-host disease (GVHD) in the absence of GVHD prophylaxis. Br J Haematol. 2019;186(5):754-766.

21. Ciceri F, Bonini C, Stanghellini MT, et al. Infusion of suicide-gene-engineered donor lymphocytes after family haploidentical haemopoietic stem-cell transplantation for leukaemia (the TK007 trial): a non-randomised phase I-II study. Lancet Oncol. 2009;10(5):489-500

22. Zhou X, Dotti G, Krance RA, et al. Inducible caspase-9 suicide gene controls adverse effects from alloreplete $\mathrm{T}$ cells after haploidentical stem cell transplantation. Blood. 2015;125(26):4103-4113.

23. Ciurea SO, Zhang MJ, Bacigalupo AA, et al. Haploidentical transplant with posttransplant cyclophosphamide vs matched unrelated donor transplant for acute myeloid leukemia. Blood. 2015;126(8):1033-1040

24. Bashey A, Zhang X, Jackson K, et al Comparison of Outcomes of Hematopoietic Cell Transplants from T-Replete Haploidentical Donors Using PostTransplantation Cyclophosphamide with 10 of 10 HLA-A, -B, -C, -DRB1, and -DOB1 Allele-Matched Unrelated Donors and HLAIdentical Sibling Donors: A Multivariable Analysis Including Disease Risk Index. Biol Blood Marrow Transplant. 2016;22(1):125133.

25. McCurdy SR, Kasamon YL, Kanakry CG, et al. Comparable composite endpoints after HLA-matched and HLA-haploidentical transplantation with post-transplantation cyclophosphamide. Haematologica. 2017; 102(2):391-400.

26. Solomon SR, Sizemore CA, Zhang X, et al. Impact of Donor Type on Outcome after Allogeneic Hematopoietic Cell Transplantation for Acute Leukemia. Biol Blood Marrow Transplant. 2016;22(10): 1816-1822.

27. Wang Y, Liu DH, Xu LP, et al. Superior graftversus-leukemia effect associated with transplantation of haploidentical compared with HLA-identical sibling donor grafts for high-risk acute leukemia: an historic comparison. Biol Blood Marrow Transplant. 2011;17(6):821-830

28. Zeidan AM, Forde PM, Symons $\mathrm{H}$, et al HLA-haploidentical donor lymphocyte infusions for patients with relapsed hematologic malignancies after related HLA-haploidentical bone marrow transplantation. Biol Blood Marrow Transplant. 2014;20(3):314-318.

29. Ghiso A, Raiola AM, Gualandi F, et al. DU after haploidentical BMT with post-transplant CY. Bone Marrow Transplant. 2015;50(1):56-61.

30. Goldsmith SR, Slade M, DiPersio JF, et al. Donor-lymphocyte infusion following haploidentical hematopoietic cell transplanta- 
tion with peripheral blood stem cell grafts and PTCy. Bone Marrow Transplant. 2017;52(12):1623-1628.

31. Yan CH, Liu DH, Liu KY, et al. Risk stratification-directed donor lymphocyte infusion could reduce relapse of standard-risk acute leukemia patients after allogeneic hematopoietic stem cell transplantation. Blood. 2012;119(14):3256-3262

32. Yan CH, Liu OF, Wu DP, et al. Prophylactic Donor Lymphocyte Infusion (DLI) Followed by Minimal Residual Disease and Graft-versus-Host Disease-Guided Multiple DLIs Could Improve Outcomes after Allogeneic Hematopoietic Stem Cell Transplantation in Patients with Refractory/Relapsed Acute Leukemia. Biol Blood Marrow Transplant. 22017;23(8):1311-1319.

33. Yan CH, Wang JZ, Liu DH, et al. Chemotherapy followed by modified donor lymphocyte infusion as a treatment for relapsed acute leukemia after haploidentical hematopoietic stem cell transplantation without in vitro T-cell depletion: superior outcomes compared with chemotherapy alone and an analysis of prognostic factors. Eur J Haematol. 2013;91(4):304-314.

34. Ma YR, Xu LP, Zhang XH, et al. Comparable post-relapse outcomes between haploidentical and matched related donor allogeneic stem cell transplantation. Bone Marrow Transplant. 2017;52(3):409-414.

35. Mo XD, Zhang XH, Xu LP, et al. Comparison of outcomes after donor lymphocyte infusion with or without prior chemotherapy for minimal residual disease in acute leukemia/myelodysplastic syndrome after allogeneic hematopoietic stem cell transplantation. Ann Hematol. 2017;96 (5):829-838.

36. Mo XD, Zhang XH, Xu LP, et al. Salvage chemotherapy followed by granulocyte colony-stimulating factor-primed donor leukocyte infusion with graft-vs.-host disease control for minimal residual disease in acute leukemia/myelodysplastic syndrome after allogeneic hematopoietic stem cell transplantation: prognostic factors and clinical outcomes. Eur J Haematol. 2016;96(3): 297-308.

37. Mo XD, Qin YZ, Zhang XH, et al. Minimal residual disease monitoring and preemptive immunotherapy in myelodysplastic syndrome after allogeneic hematopoietic stem cell transplantation. Ann Hematol. 2016;95(8):1233-1240.

38. Huang XJ, Liu DH, Liu KY, et al. Donor lymphocyte infusion for the treatment of leukemia relapse after HLAmismatched/haploidentical T-cell-replete hematopoietic stem cell transplantation. Haematologica. 2007;92(3):414-417

39. Yan CH, Xu LP, Liu DH, et al. Low-dose methotrexate may preserve a stronger antileukemic effect than that of cyclosporine after modified donor lymphocyte infusion in unmanipulated haploidentical HSCT. Clin Transplant. 2015;29(7):594-605.

40. Buckley SA, Wood BL, Othus $\mathrm{M}$, et al. Minimal residual disease prior to allogeneic hematopoietic cell transplantation in acute myeloid leukemia: a meta-analysis, Haematologica. 2017;102(5):865-873.

41. Shen Z, Gu X, Mao W, et al. Influence of pre-transplant minimal residual disease on prognosis after Allo-SCT for patients with acute lymphoblastic leukemia: systematic review and meta-analysis. BMC Cancer. 2018;18(1):755

42. Canaani J, Labopin M, Huang XJ, et al. Minimal residual disease status predicts out- come of acute myeloid leukaemia patients undergoing $\mathrm{T}$-cell replete haploidentical transplantation. An analysis from the Acute Leukaemia Working Party (ALWP) of the European Society for Blood and Marrow Transplantation (EBMT). Br J Haematol. 2018;183(3):411-420.

43. Lee HC, Saliba RM, Rondon G, et al. Mixed T Lymphocyte Chimerism after Allogeneic Hematopoietic Transplantation Is Predictive for Relapse of Acute Myeloid Leukemia and Myelodysplastic Syndromes. Biol Blood Marrow Transplant. 2015;21(11):1948-1954.

44. Koreth J, Kim HT, Nikiforow S, et al. Donor chimerism early after reduced-intensity conditioning hematopoietic stem cell transplantation predicts relapse and survival. Biol Blood Marrow Transplant. 22014;20(10): 1516-1521.

45. Solomon SR, Sizemore CA, Zhang X, et al. Preemptive DLI without withdrawal of immunosuppression to promote complete donor T-cell chimerism results in favorable outcomes for high-risk older recipients of alemtuzumab-containing reduced-intensity unrelated donor allogeneic transplant: prospective phase II trial. Bone Marrow Transplant. 2014;49(5):616-621.

46. Di Grazia C, Pozzi S, Geroldi S, et al. Wilms Tumor 1 Expression and Pre-emptive Immunotherapy in Patients with Acute Myeloid Leukemia Undergoing an Allogeneic Hemopoietic Stem Cell Transplantation. Biol Blood Marrow Transplant. 2016;22(7):1242-1246.

47. Tan Y, Du K, Luo Y, et al. Superiority of preemptive donor lymphocyte infusion based on minimal residual disease in acute leukemia patients after allogeneic hematopoietic stem cell transplantation. Transfusion. 2014;54(6):1493-1500.

48. Shah MV, Jorgensen JL, Saliba RM, et al. Early Post-Transplant Minimal Residual Disease Assessment Improves Risk Stratification in Acute Myeloid Leukemia. Biol Blood Marrow Transplant. 2018;24(7) 1514-1520.

49. Keil F, Haas OA, Fritsch G, et al. Donor leukocyte infusion for leukemic relapse after allogeneic marrow transplantation: lack of residual donor hematopoiesis predicts aplasia. Blood. 1997;89(9):3113-3117.

50. Schmid C, Schleuning M, Ledderose G, et al. Sequential regimen of chemotherapy, reduced-intensity conditioning for allogeneic stem-cell transplantation, and prophylactic donor lymphocyte transfusion in high risk acute myeloid leukemia and myelodysplastic syndrome. J Clin Oncol. 2005;23(24): 5675-5687.

51. Tsirigotis P, Liga M, Gkirkas K, et al. Lowdose alemtuzumab for GvHD prevention followed by prophylactic donor lymphocyte infusions in high-risk leukemia. Bone Marrow Transplant. 2017:52(3):445-451.

52. Maschan M, Blagov S, Shelikhova L, et al. Low-dose donor memory T-cell infusion after TCR alpha/beta depleted unrelated and haploidentical transplantation: results of a pilot trial. Bone Marrow Transplant. 2018;53(3):264-273.

53. Schmid C, Labopin M, Schaap N, et al. Prophylactic donor lymphocyte infusion after allogeneic stem cell transplantation in acute leukaemia - a matched pair analysis by the Acute Leukaemia Working Party of EBMT. Br J Haematol. 2018;184(5):782-787.

54. Gao XN, Lin J, Wang SH, et al. Donor lymphocyte infusion for prevention of relapse after unmanipulated haploidentical PBSCT for very high-risk hematologic malignancies.
Ann Hematol. 2018;98(1):185-193

55. Jaiswal SR, Zaman S, Nedunchezhian M, et al. CD56-enriched donor cell infusion after post-transplantation cyclophosphamide for haploidentical transplantation of advanced myeloid malignancies is associated with prompt reconstitution of mature natural killer cells and regulatory $T$ cells with reduced incidence of acute graft versus host disease: A pilot study. Cytotherapy. 2017;19(4):531-542.

56. Jaiswal SR, Zaman S, Chakrabarti A, et al Improved Outcome of Refractory/Relapsed Acute Myeloid Leukemia after PostTransplantation Cyclophosphamide-Based Haploidentical Transplantation with Myeloablative Conditioning and Early Prophylactic Granulocyte ColonyStimulating Factor-Mobilized Donor Lymphocyte Infusions. Biol Blood Marrow Transplant. 2016;22(10):1867-1873.

57. Yan CH, Wang Y, Wang JZ, et al. Minimal residual disease- and graft-vs.-host diseaseguided multiple consolidation chemotherapy and donor lymphocyte infusion prevent second acute leukemia relapse after allotransplant. J Hematol Oncol. 2016;9(1):87.

58. Nagler A, Ackerstein A, Or R, et al. Adoptive immunotherapy with haploidentical allogeneic peripheral blood lymphocytes following autologous bone marrow transplantation. Exp Hematol. 2000;28(11):1225 1231

59. Call SK, Kasow KA, Barfield R, et al. Total and active rabbit antithymocyte globulin (rATG; Thymoglobulin) pharmacokinetics in pediatric patients undergoing unrelated donor bone marrow transplantation. Biol Blood Marrow Transplant. 2009;15(2):274278.

60. Gilman AL, Leung W, Cowan MJ, et al Donor lymphocyte infusion and methotrexate for immune recovery after T-cell depleted haploidentical transplantation. Am Hematol. 2018;93(2):169-178.

61. Martelli MF, Di Ianni M, Ruggeri L, et al HLA-haploidentical transplantation with regulatory and conventional $\mathrm{T}$-cell adoptive immunotherapy prevents acute leukemia relapse. Blood. 2014;124(4):638-644

62. Dodero A, Carniti C, Raganato A, et al Haploidentical stem cell transplantation after a reduced-intensity conditioning regimen for the treatment of advanced hematologic malignancies: posttransplantation CD8-depleted donor lymphocyte infusions contribute to improve $\mathrm{T}$-cell recovery. Blood. 2009;113(19):4771-4779.

63. Perruccio K, Tosti A, Burchielli E, et al Transferring functional immune responses to pathogens after haploidentical hematopoietic transplantation. Blood. 2005;106(13):4397-4406.

64. Edinger M, Hoffmann P, Ermann J, et al CD4+CD25+ regulatory $\mathrm{T}$ cells preserve graft-versus-tumor activity while inhibiting graft-versus-host disease after bone marrow transplantation. Nat Med. 2003;9(9):11441150

65. Lewalle P, Triffet A, Delforge A, et al. Donor lymphocyte infusions in adult haploidentical transplant: a dose finding study. Bone Marrow Transplant. 2003;31(1):39-44

66. Wang Y, Liu DH, Xu LP, et al. Prevention of relapse using granulocyte CSF-primed PBPCs following HLA-mismatched/haploidentical, T-cell-replete hematopoietic SCT in patients with advanced-stage acute leukemia: a retrospective risk-factor analysis. Bone Marrow Transplant. 2012;47(8): 1099-1104. 
67. Cauchois R, Castagna L, Pagliardini T, et al. Prophylactic donor lymphocyte infusions after haploidentical haematopoietic stem cell transplantation for high risk haematological malignancies: a retrospective bicentric analysis of serial infusions of increasing doses of CD3(+) cells. Br J Haematol. 2019;185(3):570-573.

68. Dazzi F, Szydlo RM, Cross NC, et al. Durability of responses following donor lymphocyte infusions for patients who relapse after allogeneic stem cell transplantation for chronic myeloid leukemia. Blood. 2000;96(8):2712-2716.

69. Yan CH, Liu DH, Xu LP, et al. Modified donor lymphocyte infusion-associated acute graft-versus-host disease after haploidentical T-cell-replete hematopoietic stem cell transplantation: incidence and risk factors. Clin Transplant. 2012;26(6):868-876

70. Jun HX, Jun CY, Yu ZX. In vivo induction of T-cell hyporesponsiveness and alteration of immunological cells of bone marrow grafts using granulocyte colony-stimulating factor. Haematologica. 2004:89(12):1517-1524.

71. Wen Q, Kong Y, Zhao HY, et al. G-CSFinduced macrophage polarization and mobilization may prevent acute graft-versus-host disease after allogeneic hematopoietic stem cell transplantation. Bone Marrow Transplant. 2019;54(9):1419-1433.

72. Chen SH, Li X, Huang XJ. Effect of recombinant human granulocyte colony-stimulating factor on T-lymphocyte function and the mechanism of this effect. Int J Hematol. 2004;79(2):178-184.

73. Huang XJ, Chang YJ, Zhao XY. Maintaining hyporesponsiveness and polarization potential of T cells after in vitro mixture of G-CSF mobilized peripheral blood grafts and GCSF primed bone marrow grafts in different proportions. Transpl Immunol. 2007;17(3): 193-197.

74. Gómez-De León A, Colunga Pedraza PR, Garcia-Camarillo DE, et al. Donor Lymphocyte Infusion from G-CSF-Primed, Unmanipulated Whole Blood Is Safe and Improves Chimerism in HLA-Matched and Haploidentical Transplantation. Blood. 2018;132(Suppl 1):4602.

75. Bracci L, Moschella F, Sestili P, et al. Cyclophosphamide enhances the antitumor efficacy of adoptively transferred immune cells through the induction of cytokine expression, B-cell and T-cell homeostatic proliferation, and specific tumor infiltration. Clin Cancer Res. 2007;13(2 Pt 1):644-653.

76. He F, Warlick E, Miller JS, et al. Lymphodepleting chemotherapy with donor lymphocyte infusion post-allogeneic HCT for hematological malignancies is associated with severe, but therapy-responsive aGvHD. Bone Marrow Transplant. 2016:51(8): 1107-1112.

77. Goodyear O, Agathanggelou A, NovitzkyBasso I, et al. Induction of a CD8+ T-cell response to the MAGE cancer testis antigen by combined treatment with azacitidine and sodium valproate in patients with acute myeloid leukemia and myelodysplasia. Blood. 2010;116(11):1908-1918.

78. Guillaume T, Yakoub-Agha I, Tabrizi R, et al. Prospective Phase II Study of Prophylactic Azacitidine and Donor Lymphocyte Infusions Following Allogeneic Hematopoietic Stem Cell Transplantation for High Risk Acute Myeloid Leukemia and Myelodysplastic Syndrome. Blood. 2016; 128(22):1162

79. Schroeder T, Rautenberg C, Haas R, Kobbe G. Hypomethylating agents after allogeneic blood stem cell transplantation. Stem Cell Investig. 2016;3:84.

80. Schroeder T, Czibere A, Platzbecker U, et al Azacitidine and donor lymphocyte infusions as first salvage therapy for relapse of AML or MDS after allogeneic stem cell transplantation. Leukemia. 2013;27(6):12291235 .

81. Schroeder T, Rautenberg C, Kruger W, et al. Treatment of relapsed AML and MDS after allogeneic stem cell transplantation with decitabine and DLI-a retrospective multicenter analysis on behalf of the German Cooperative Transplant Study Group. Ann Hematol. 2018;97(2):335-342

82. Bazarbachi A, Labopin M, Battipaglia G, et al. Allogeneic Stem Cell Transplantation for FLT3-Mutated Acute Myeloid Leukemia: In vivo T-Cell Depletion and Posttransplant Sorafenib Maintenance Improve Survival. A Retrospective Acute Leukemia Working Party-European Society for Blood and Marrow Transplant Study. Clin Hematol Int. 2019;1(1):58-74.

83. Canaani J. Management of AML Beyond " 3 + 7" in 2019. Clin Hematol Int. 2019;1:10-18.

84. Schmidt SA, Holter Chakrabarty J, Liu Y, et al. Tyrosine Kinase Inhibitors with or without Donor Lymphocyte Infusion Continue to Provide Long-Term Survival after Relapse of Chronic Myeloid Leukemia Following Hematopoietic Cell Transplantation. Blood. 2018;132(Suppl 1):704.

85. Vago L, Perna SK, Zanussi M, et al. Loss of mismatched HLA in leukemia after stem-cell transplantation. N Engl J Med. 2009;361 (5):478-488.

86. Crucitti L, Crocchiolo R, Toffalori C, et al. Incidence, risk factors and clinical outcome of leukemia relapses with loss of the mismatched HLA after partially incompatible hematopoietic stem cell transplantation. Leukemia. 2015;29(5):1143-1152.

87. Imus PH, Blackford AL, Bettinotti M, et al. Major Histocompatibility Mismatch and Donor Choice for Second Allogeneic Bone Marrow Transplantation. Biol Blood Marrow Transplant. 2017;23(11):18871894

88. McCurdy SR, Iglehart BS, Batista DA, et al. Loss of the Mismatched Human Leukocyte Antigen Haplotype in Two Acute Myelogenous Leukemia Relapses after Haploidentical Bone Marrow Transplantation with Posttransplantation Cyclophosphamide. Leukemia. 2016;30(10): 2102-2106.

89. Russo A, Oliveira G, Berglund S, et al. NK cell recovery after haploidentical HSCT with posttransplant cyclophosphamide: dynamics and clinical implications. Blood. 2018;131(2):247-262

90. Jaiswal SR, Bhakuni P, Bharadwaj P, et al. CTLA4Ig Primed Donor Lymphocyte Infusions (DLI): A Novel Approach to Natural Killer Cell Immunotherapy Following Haploidentical PBSC Transplantation for Advanced Hematological Malignancies. Blood. 2017; 130(Suppl 1):4468

91. Ciurea SO, Schafer JR, Bassett R, et al. Phase 1 clinical trial using mbIL21 ex vivo-expanded donor-derived NK cells after haploidentical transplantation. Blood. 2017;130(16); 1857-1868.

92. Szczepanski MJ, Szainik M, Welsh A, et al Interleukin-15 enhances natural killer cell cytotoxicity in patients with acute myeloid leukemia by upregulating the activating NK cell receptors. Cancer Immunol Immunother. 2010;59(1):73-79.
93. Romee R, Rosario M, Berrien-Elliott MM, et al. Cytokine-induced memory-like natural killer cells exhibit enhanced responses against myeloid leukemia. Sci Transl Med. 2016;8(357):357ra123

94. Langenkamp U, Siegler U, Jorger S, et al. Human acute myeloid leukemia CD34+CD38- stem cells are susceptible to allorecognition and lysis by single KIRexpressing natural killer cells. Haematologica. 2009;94(11):1590-1594.

95. Roy D-C, Walker I, Maertens J, et al. Efficacy and Safety of a Single Dose of Donor Lymphocytes Depleted of Alloreactive T-Cells (ATIR101) Following TCell-Depleted Haploidentical HSCT: A Pooled Analysis of Two Phase II Studies. Blood. 2018;132(Suppl 1):120.

96. Davies JK, Brennan LL, Wingard JR, et al, Infusion of Alloanergized Donor Lymphocytes after CD34-selected Haploidentical Myeloablative Hematopoietic Stem Cell Transplantation. Clin Cancer Res. 2018;24(17):4098-4109.

97. Di Stasi A, Tey SK, Dotti G, et al. Inducible apoptosis as a safety switch for adoptive cell therapy. N Engl J Med. 2011;365(18):16731683.

98. Merli P, Bertaina V, Galaverna F, et al. Donor T Cells Genetically Modified with a Novel Suicide Gene (inducible Caspase 9, iC9) Expand and Persist over Time after PostAllograft Infusion in Patients Given $\alpha \beta$ TCell and B-Cell Depleted HLAHaploidentical Allogeneic Stem Cell Transplantation $\quad(\alpha \beta$ haplo-HSCT) Contributing to Accelerate Immune Recovery. Blood. 2017;130(Suppl 1):211.

99. Research CfDEa. Approved Drugs - FDA approves tisagenlecleucel for B-cell ALL and tocilizumab for cytokine release syndrome. 2018

100. Neelapu SS, Locke FL, Bartlett NL, et al Axicabtagene Ciloleucel CAR T-Cell Therapy in Refractory Large B-Cell Lymphoma. N Engl J Med. 2017;377(26): 2531-2544.

101. Kebriaei P, Ciurea SO, Huls MH, et al. PreEmptive Donor Lymphocyte Infusion with CD19-Directed, CAR-Modified T Cells Infused after Allogeneic Hematopoietic Cell Transplantation for Patients with Advanced CD19<sup $>+</$ sup $>$ Malignancies. Blood. 2015;126(23):862.

102. Liu J, Zhong JF, Zhang X, et al. Allogeneic CD19-CAR-T cell infusion after allogeneic hematopoietic stem cell transplantation in $\mathrm{B}$ cell malignancies. J Hematol Oncol. 2017;10 (1):35

103. Radestad E, Wikell H, Engstrom M, et al Alpha/beta T-cell depleted grafts as an immunological booster to treat graft failure after hematopoietic stem cell transplantation with HLA-matched related and unrelated donors. J Immunol Res. 2014;2014: 578741.

104. Durer C, Durer S, Shafqat M, et al Concomitant Use of Blinatumomab and Donor Lymphocyte Infusion for PostTransplant Relapsed CD19 Positive Acute Lymphoblastic Leukemia. Syst Rev. 2018;132:5742

105. Christopher MJ, Petti AA, Rettig MP, et al Immune Escape of Relapsed AML Cells after Allogeneic Transplantation. N Engl J Med. 2018:379(24):2330-2341

106. Or R, Hadar E, Bitan M, et al. Safety and efficacy of donor lymphocyte infusions following mismatched stem cell transplantation. Biol Blood Marrow Transplant. 2006;12(12): 1295-1301. 